\title{
Cyclophilins A and B Oppositely Regulate Renal Tubular Epithelial Phenotype
}

Eduard Sarró ${ }^{1 \#}$, Mónica Durán ${ }^{1}$, Ana Rico ${ }^{1}$, Anthony J. Croatt ${ }^{2}$, Karl A. Nath ${ }^{2}$, Salcedo Maria Teresa $^{3}$, Justin H. Gundelach ${ }^{4}$, Daniel Batlle ${ }^{5,}$ Richard J. Bram ${ }^{4,6}$, Anna Meseguer ${ }^{1,7,8 \#}$.

Affiliations: ${ }^{1}$ Renal Physiopathology Group, CIBBIM-Nanomedicine, Vall d'Hebron Research Institute, Barcelona, Spain. ${ }^{2}$ Division of Nephrology and Hypertension and Departments of Medicine, Mayo Clinic, Rochester, Minnesota, USA. ${ }^{3}$ Department of Pathology, Hospital Universitari Vall d'Hebron, Barcelona, Spain. ${ }^{4}$ Department of Pediatric and Adolescent Medicine, College of Medicine, Mayo Clinic, Rochester, Minnesota, USA. ${ }^{5}$ Division of Nephrology and Hypertension, Northwestern University Feinberg School of Medicine, Chicago, Illinois, USA. ${ }^{6}$ Department of Immunology, College of Medicine, Mayo Clinic, Rochester, Minnesota, USA. ${ }^{7}$ Departament de Bioquímica i Biologia Molecular, Unitat de Bioquímica de Medicina, Universitat Autònoma de Barcelona, Bellaterra; Spain. ${ }^{8}$ Red de Investigación Renal (REDINREN), Instituto Carlos III-FEDER, Madrid, Spain.

\# Corresponding authors:

Eduard Sarró: eduard.sarro@vhir.org

Anna Meseguer: ana.meseguer@vhir.org

Renal Physiopathology Group, CIBBIM-Nanomedicine, Vall d'Hebron Research Institute, Passeig Vall d'Hebron 119-129, 08035 Barcelona, Spain.

Telephone number: +34 934894070; fax number: +34 934894015;

Running headline: CypA and B regulate epithelial phenotype

Keywords: Cyclophilins, epithelial phenotype, Slug, Snail, TGF $\beta$, UUO, fibrosis

Character count: 


\section{Abstract}

2

Cyclophilins (Сур) are peptidil-prolyl-isomerases and the intracellular receptors for the immunosuppressant Cyclosporine-A (CsA), which produces epithelial-mesenchymal-transition

$4 \quad($ EMT) and renal tubule-interstitial fibrosis. Since CsA inhibits Cyp enzymatic activity, we

5 hypothesized that Cyp could be involved in EMT and fibrosis. Here, we demonstrate that CypB

6 is a critical regulator of tubule epithelial cell plasticity on the basis that: i) CypB silencing caused

7 epithelial differentiation in proximal tubule-derived HK-2 cells, ii) CypB silencing prevented

8 TGFß-induced EMT in HK-2, and iii) CypB knockdown mice exhibited reduced UUO-induced

9 inflammation and kidney fibrosis. By contrast, silencing of CypA induces a more undifferentiated

10 phenotype and favors TGF $\beta$ effects. EMT mediators Slug and Snail were up-regulated in CypA-

11 silenced cells, while in CypB silencing, Slug, but not Snail, was down-regulated; thus,

12 reinforcing the role of Slug in kidney fibrosis. CypA regulates Slug through its PPlase activity

13 whereas CypB depends on its ER location, where interacts with calreticulin, a calcium

14 modulator which is involved in TGF $\beta$ signaling. In conclusion, this work uncovers new roles for

15 CypA and CypB in modulating proximal tubular cell plasticity. 


\section{Introduction}

Kidney fibrosis is the principal process underlying the progression of chronic kidney disease (CKD) to end stage renal disease (ESRD). Since specific therapies to prevent, slow down, or reverse fibrosis are severely lacking (1), understanding the complex molecular mechanisms and cellular mediators of kidney fibrosis could offer new therapeutic avenues to prevent the loss of kidney function (2).

Maladaptive repair due to repeated or sustained injury to the proximal tubule epithelial cells (PTC) has proved to be sufficient to induce CKD and fibrosis (3). Injured PTC, which drive injury and inflammation by releasing pro-fibrotic factors, in particular TGF $\beta$ (4), and by producing inflammatory cytokines, including $\operatorname{TNFa}(5,6)$, have been identified as a major player in fibrosis. A very marked and relevant feature of kidney fibrosis is the transition of tubular epithelial cells into cells with mesenchymal features, a so-called epithelial-mesenchymal transition (EMT) (7). This switch in cell differentiation and behavior is mediated by transcription factors, including Snail (Snail1), Slug (Snail2), zinc-finger E-box-binding homeobox (ZEB)1/2 and Twist1/2, which negatively regulate E-cadherin expression through their recognition of common E-box sequences in the E-cadherin promoter $(8,9)$. Down-regulation of E-cadherin is the hallmark of EMT to reinforce the destabilization of adherens junctions in the epithelial barrier. In vivo, it has been proved that Snail is responsible for the partial EMT program (EMT2) that leads to dedifferentiation of renal epithelial cells and promotes kidney fibrosis $(10,11)$. Damaged epithelial cells undergoing EMT2 remain confined to the tissue without engaging in the delamination and invasion programs occurring in cancer (EMT3) (11).

The potent immunosuppressant Cyclosporine A (CsA) produces severe renal tubuleinterstitial fibrosis that limit the drug's clinical use (12). In vitro, CsA treatment of PTC induces a dose-dependent release of TGF- $\beta$, EMT events and increased expression of Snail (13), as well as, production of pro-inflammatory cytokines (14). Intracellularly, CsA binds to cyclophilins, a family of ubiquitous and highly conserved proteins that accelerate protein folding by catalyzing the cis-trans isomerization of proline residue $(15,16)$. CsA binding to cyclophilins inhibits their peptidyl-prolyl cis-trans isomerase (PPlase) activity. Cyclophilins do also play a prominent role as chaperones by mediating protein trafficking, protein-protein interactions, and as scaffolding 
proteins for assembly of macromolecular complexes (17). In humans, cyclophilins A (CypA) and $B($ СypB), the best characterized members of the family, are located in the cytosol and in the endoplasmic reticulum (ER), respectively (17). CypB controls ER homeostasis by participating in the protein quality control process in the ER (18) and is released to the extracellular media in the presence of CsA (19). Moreover, CypA and CypB can also be released in response to inflammatory stimuli and elevated circulating levels for both of them have been reported in different inflammatory diseases (20-22). They contribute to the inflammatory responses via their potent chemotactic properties for various immune cells $(23,24)$, which is mediated by the signaling receptor CD147 on target cells (25). In the kidney, CypB was found to be the interacting partner of KAP, a protein exclusively expressed in proximal tubule cells of the kidney that protects against CsA toxicity $(26,27)$, as well as interacting with sodium-potassium ATPase, being required for pump activity in proximal tubule cells of the kidney (28). on cyclophilins, the idea that the latest could be critical in the development of EMT and kidney fibrosis is entirely plausible. To investigate the potential involvement of cyclophilins in the regulation of the epithelial phenotype, we studied the effects of CypA and CypB silencing in human proximal tubule epithelial cells. We observed that CypA and CypB silencing exert opposite effects on the phenotype of proximal tubular cells by promoting or haltering, respectively, EMT processes through distinctly modulating the Snail family of epithelial repressors. Moreover, we also showed a marked attenuation of the molecular changes associated with inflammation and fibrosis in the kidneys of CypB KO mice subjected to unilateral ureteral obstruction (UUO). Results from this study pinpoint CypB as a potential therapeutic target to prevent fibrosis. 


\section{Results}

\section{CypB and CypA silencing differentially affects epithelial phenotype of cultured}

PTC

To investigate the potential involvement of cyclophilins in the regulation of the epithelial phenotype, we silenced CypA and CypB in HK-2 cells, a widely characterized proximal tubular epithelial cell line retaining a phenotype indicative of well-differentiated PTC (Fig. 1A). Since PTC in culture progressively acquire epithelial features upon reaching confluence, we first analyzed the expression levels of the epithelial markers E-cadherin (adherens junctions), ZO-1 and occludin (tight junctions) and keratin (intermediate filaments) at 2, 5 and 10 days post seeding, considering that cells reach confluence by the second day. We observed that all epithelial markers increased along days of culture (Fig. 1B). Five days post seeding was selected for further experiments. Our results show that CypB silencing greatly increased Ecadherin and occludin expression and to a lesser extent ZO-1 and keratin (Fig. 1C). By contrast CypA silencing reduced occludin, ZO-1 and keratin levels. Those results were also observed at the mRNA level (Fig. 1D). As shown in Figure 1E, the increase in E-cadherin expression observed in CypB-silenced cells correlated with augmented E-cadherin levels in the plasma membrane, suggesting a concomitant gain in E-cadherin functionality. Since a stimulatory role for E-cadherin in proliferation has been described (29), we explored the effect of CypB knockdown on HK-2 proliferation. In accordance with E-cadherin levels, cells lacking CypB showed higher proliferation indices than control cells, whereas CypA silencing had no significant effect (Fig 1F). By contrast, we observed that CypA silencing reduced transepithelial electric resistance (TER) and increased FITC-labeled Dextran permeability (Fig. 1G and 1H, respectively), which correlated with the downregulation in ZO-1 and occludin levels observed in CypA-silenced cells.

To further characterize the effects of cyclophilin silencing in epithelial differentiation, we analyzed the activities of the proximal tubule brush border enzymes alkaline phosphatase (AP), dipeptidyl peptidase-IV (DPP-IV) and gamma-glutamyltransferase (GGT) as recognized markers of epithelial differentiation. Aligned with the above results, AP activity was markedly enhanced in cells lacking CypB, with maximal levels already achieved after two days of culture 
(Fig. 1I). By contrast, DPPIV activity stayed below control levels (Fig. 1J) and GGT activity behaved as in controls (Fig. 1K). Diminished DPPIV activity might not be detrimental to the renal epithelial cells phenotype since it has been reported that DPPIV inhibitors block TGF $\beta$ signaling, thus protecting against renal fibrosis $(30,31)$. In CypA-silenced cells AP activity remained permanently low (Fig. 1I), DPPIV activity behaved as in control cells (Fig. 1J) and GGT activity increased over time but remained below control levels (Fig. 1K).

The temporal expression pattern of epithelial markers is tightly regulated by epithelial repressors, including the transcription factors Snail (Snail1), Slug (Snail2), zinc-finger E-boxbinding homeobox (ZEB)1/2, and Twist1/2 $(8,9)$. Our results show that in HK-2 cells CypA silencing increased Slug mRNA levels and to a lesser extent those of Snail (Fig. 1L). Interestingly, CypB silencing also slightly increased the mRNA levels of Snail, but strongly reduced those of Slug (Fig. 1L). By contrast, neither CypA nor CypB silencing had any significant effect on Twist1 and Zeb1 mRNA levels (Fig. 1L). To corroborate these results, we analyzed the protein levels of Slug and Snail. Our results show that Slug and Snail expression progressively decreased from day 2 to 10 (Fig. 1M), correlating with the augmented epithelial marker expression observed in Figure 1B. Moreover, at 5 days post-seeding, we observed that Slug protein levels were increased in CypA-silenced cells while they were undetectable in CypB-silenced cells (Fig. 1N). Both CypA and CypB increased Snail protein levels to a higher extent than that observed at the mRNA level, suggesting additional regulatory mechanisms besides transcriptional modulation (Fig. 1N).

In order to corroborate these results, we silenced СypB and СурА in another proximal tubule derived cell line (RPTEC) (Fig S1). Our results show that, as in HK-2 cells, RPTEC cells lacking СypВ presented reduced slug expression and higher Snail levels. E-cadherin upregulation in CypB silenced cells was less appreciable in RPTEC cells, mostly due to the higher basal expression of this protein in comparison with HK-2 cells.

Taken together, these results indicate that while CypB is necessary for Slug expression, CypA acts as a repressor of both Slug and Snail. Moreover, our results also indicate that, at least in CypB-silenced cells, loss of Slug rather than gain of Snail prevail in the regulation of epithelial markers. 


\section{Cyclophilin A and B exert divergent effects on TGF $\beta$ action on epithelial cells}

TGF $\beta$ has been widely regarded as a primary factor driving renal fibrosis (4). In vitro,

TGF $\beta$ treatment alone can induce proximal tubule cells to undergo an epithelial-to-

mesenchymal transition (EMT) $(4,32)$. Considering the results shown in Figure 1, we decided to investigate the effects of CypB and CypA silencing on TGF $\beta$ signaling. In HK-2 cells, TGF $\beta$ induced an EMT-like process demonstrated by a gradual decrease of E-cadherin and occludin expression and an increase of Fibronectin levels that were not related with changes in CypA or CypB levels (Fig. 2A). Reminiscent of what happens in basal conditions, it is of note that CypB silencing partially prevented TGF $\beta$-induced EMT by maintaining E-cadherin and occludin levels closer to control and reducing fibronectin expression (Fig. 2B). In addition, CypA silencing enhanced TGF $\beta$-induced EMT by further decreasing E-cadherin and occludin levels and increasing those of fibronectin. Loss of E-cadherin and occludin expression after TGF $\beta$ treatment was consistent with a switch from a well defined monolayer with cobblestone morphology of control cells to formation of cell aggregates containing a combination of poorlyinterconnected rounded cells and spindle-shaped cells with filopodia (shCon panels of Fig. 2C) and increased cell-to-substrate adhesiveness (shCon bar of Fig. 2D) in treated control cells. Silencing of CypB almost completely prevented TGF $\beta$-induced morphological changes (Fig. 2C) and strongly hampered the increase cell-to-substrate adhesion induced by TGF $\beta$ treatment, while CypA silencing had no effect (Fig. 2D).

Accordingly, we analyzed whether the distinct phenotypical changes observed in TGF $\beta$-treated CypA and CypB-silenced cells could be related to Smad-dependent regulation of Slug and Snail levels. Treatment of HK-2 cells with TGF $\beta$ induced a time-dependent expression of Slug and Snail that was preceded by Smad2/3 activation (Fig. 2E). Silencing of CypB prevented TGFßinduced slug expression and enhanced Snail expression, while silencing of CypA increased both Slug and Snail levels (Fig. 2F left). Again, these results support a predominant effect of Slug downregulation over Snail upregulation in the TGF $\beta$-induced morphological changes observed in CypB-silenced cells. Interestingly, cyclophilin modulation of Slug and Snail occurred 
without changes in TGF $\beta$-induced phosphorylation of Smad2/3 or in the expression levels of Smad4. Although to a lesser extent, these changes were also observed at the transcriptional level (Fig. 2F right). Slug and Snail proteins have a rapid turnover that is regulated by ubiquitinmediated proteasomal degradation. To further demonstrate that СypB silencing diminished Slug expression primarily through transcriptional effects, cells were pre-treated with the proteasome inhibitor MG132 before treatment with TGF $\beta$. As depicted in Figure 2G, treatment with MG132 alone increased Slug levels well over those of TGF $\beta$ induction. This increase was clearly reduced in CypB silenced cells, an effect even more evident after TGF $\beta$ induction, supporting the concept that CypB modulates Slug at the mRNA level.

In addition to phosphorylation, TGF $\beta$-induced Smad2/3 signaling is also regulated by other mechanisms such as nuclear translocation or through the action of inhibitory Smad7 and Smad6, which negatively regulate TGF $\beta$ signaling by establishing an autoregulatory negative feedback loop (33). First we analyzed Smad3 and Smad2 translocation to the nucleus in CypB and CypA-silenced cells after TGF $\beta$ treatment (Figure $2 \mathrm{H}$ ). Our results showed that neither CypB nor CypA silencing affected TGF $\beta$-induced Smad2/3 translocation. We next analyzed the expression levels of Smad7 and 6, and of Snon, a transcriptional repressor of Smad2/3 regulated genes. As shown in Figure 21, Smad7, Smad6, and Snon levels were transcriptionally induced by TGF $\beta$ treatment. Interestingly, all three genes were upregulated in CypB-silenced cells in both untreated and TGF $\beta$ treated cells and slightly reduced in CypA-silenced cells after TGF $\beta$ treatment.

In order to explore the mechanisms by which CypB regulates inhibitory Smads, we analyzed the levels of BMPs 2 and 7, which counteract TGF $\beta$-induced EMT in a Smad dependent way. We were unable to detect BMP7 mRNA in HK2 cells despite the use of multiple different probes. By contrast, we detected BMP2, which was upregulated in CypB silenced cells (Fig. 2l). 
Proximal tubular cells (PTC) actively contribute to the production of inflammatory

mediators $(5,34,35)$, thereby participating to dynamic interplay between fibrosis and inflammation. It has also been reported that NFKB, which plays a key role in inflammation, upregulates Snail both at the transcriptional level and stabilizing Snail protein (36). We hypothesized a putative role of cyclophilins on these processes and analyzed the promoter activity of NFKB in CypA and CypB silenced cells in basal conditions and upon activation by cotransfection with p65 subunit. Our results show no significant differences in NFKB promoter activity between control and silenced cells in basal conditions (Fig. 3A). However, when NFKB was activated by p65 cotransfection, we observed that the promoter activity was strongly increased in CypA-silenced cells and considerably decreased in CypB-silenced cells.

Because it has been described a role for extracellular cyclophilins in inflammation (37), we next aimed to explore whether cyclophilin regulation of Slug and Snail levels could be related to modulation of this inflammatory pathway in an autocrine manner. Figure 3B shows that both CypB and CypA were progressively secreted into the media of HK-2 cells and that secretion was unaffected by TGF $\beta$ treatment. To explore whether extracellular CypB could be supporting Slug expression, we blocked CypB secretion with Brefeldin-A (Bf-A) to inhibit protein transport from the endoplasmic reticulum to the Golgi apparatus as well as with Cyclosporine-A (CsA) to further induce CypB secretion. Our results show that Bf-A blocked both basal and CsAinduced CypB secretion (Fig. 3C). By contrast, neither CsA nor Bf-A had any effect on CypA secretion. This shows that СурА and СурB are secreted to the extracellular media through different mechanisms. Bf-A pre-treatment prevented basal and TGF $\beta$-induced Slug expression (Fig. 3D) but, contrarily than CypB silencing, also reduced Snail expression and Smad3 and Smad2 activation. To further explore the involvement of extracellular cyclophilins, we knocked down CD147, which is, to our knowledge, the only known receptor for extracellular CypB and CypA. Our results show that CD147 silencing increased, rather than decreased, Slug and Snail levels, thereby resembling our findings in CypA but not in CypB-silenced cells (Fig. 3E). Finally, and to further investigate whether extracellular CypB could be modulating Slug levels in a CD147 independent manner, CypB-silenced cells were treated with increasing doses of recombinant CypB. Our results show that exogenous CypB was unable to restore Slug expression or downregulate Snail levels to levels of non-silenced cells (Fig. 3F). ERK1/2 
phosphorylation was used as a control of CypB treatment effectiveness (38). Taken together these results argue against and autocrine loop in Slug modulation by СурВ. expression by CypB and CypA, we re-introduced mutated forms of CypB and CypA lacking PPlase activity or, in the case of CypB, its $\mathrm{N}$-terminal signal peptide, in the corresponding silenced-cells (Fig. 4A). To do so, we first generated a shRNA-resistant wild-type CypB (wt CypB) by introducing silent mutations into the shRNA-targeted sequence for CypB to allow its escape from degradation by the RISC complex. ShRNA against CypA was directed to the 3'UTR, and thus reintroduction of a wild-type form of CypA (wt CypA) did not require any further modification. Re-introduction of the wt forms served as rescue experiments to validate that the effects observed in silenced cells were not due to off-targets effects. Over these constructs, we mutated critical residues for PPlase activity ( $\triangle \mathrm{PPI}$ mutants) in both CypB (R62A) and CypA (R55A), and separately deleted the signal peptide $\left(\Delta\left(\mathrm{K} 2 \_\mathrm{A} 25\right)\right)$ directing CypB to the endoplasmic reticulum ( $\triangle E \mathrm{E}$ mutant). Finally, an HA-tag was added at the C-terminus of each cDNA. All these constructs were stably transduced into cyclophilin-silenced cells using lentiviral particles and selected with a different antibiotic than the one used for silencing selection. Western blot assays demonstrated that the wild-type and the mutant forms of both CypA and CypB were successfully reintroduced in the corresponding silenced cells (Fig. 4B and 4E, respectively). Moreover, by using confocal IF we observed that both wt and $\triangle \mathrm{PPI}$ forms of CypB staining correlated with ER location, as determined by the ER transmembrane protein calnexin (CNX), and were successfully secreted to the extracellular medium (Fig. 4F and 4E, SN panel), while CypB $\triangle \mathrm{ER}$ did not (Fig. 4F and 4E, SN panel). Slug and Snail levels to those of control cells, while reintroduction of the $\triangle \mathrm{PPI}$ mutant of CypA failed to do so, indicating that the PPlase activity of CypA is necessary to maintain Slug and 
242 the other hand, reintroduction of CypB-wt into CypB-silenced cells increased Slug levels almost

243 to control levels while that of the $\triangle \mathrm{PPI}$ mutant not only rescued but increased Slug levels over

244 those of control cells (Fig. 4G). Finally, reintroduction of the $\Delta$ ER mutant failed to restore Slug

245 levels. Again, these effects were observed in the presence of TGF $\beta$ (Fig. 4H). These results

246 reinforce the idea that CypB is required for Slug expression and that its presence in the ER but

247 not its PPlase activity is required for this effect.

As shown above, ER location of CypB is mandatory for Slug expression. Since it has been previously described that CypB interacts with the calcium-related ER chaperones calreticulin (CRT) and calnexin (CNX) (39), and CRT has been involved in the regulation of Slug expression (40-42), we analyzed whether the modulatory effects of CypB on Slug levels could be related to changes in CRT/CNX localization or altered interaction with these chaperones. Figure 5A shows that CRT and CNX colocalized discretely, fitting with their ER luminal and transmembrane location, respectively. We also observed that localization of CRT and CNX was unaffected by either CypA or CypB silencing. Next, wild type, PPlase and signal peptide defective mutants of СурB were re-introduced in CypB-silenced cells and immunoprecipitated with HA antibody. As shown in Figure 5B, CRT immunoprecipitated with CypB wt and to a higher extent with CypB $\triangle \mathrm{PPI}$ but no interaction was detected with the CypB $\Delta \mathrm{ER}$ mutant. Interestingly, this interaction pattern paralleled that of Slug expression shown in Figure 4G. By contrast, we detected CNX interaction with the CypB $\Delta \mathrm{ER}$ mutant but not with the wt form. Although a strong interaction of $\mathrm{CNX}$ with $\mathrm{CypB} \triangle \mathrm{PPI}$ was also observed, it is worth mentioning that $\mathrm{CNX}$ levels were increased in CypB $\triangle \mathrm{PPI}$ cell extracts (input). Taken together these results suggest that a CypB-CRT complex rather than a CypB-CNX complex could be mandatory for CypB regulation of Slug. They also indicate that interaction with either CRT or CNX depends on CypB location. storage and release. To ascertain if Slug downregulation in CypB silenced cells could be due to ER-related calcium signaling, we analyzed whether Slug and Snail levels were modulated by 
271 alterations of ER calcium stores. ER calcium pools were depleted by exposing cells to

272 thapsigargin or ionomycin (Fig. 5C). Our results show that basal Slug levels were increased by

273 ionomycin but suppressed by thapsigargin, while Snail levels were downregulated by both.

274 These effects were also observed in the presence of TGF $\beta$ (Fig. 5D). Interestingly, ionomycin

275 was unable to induce Slug expression in CypB-silenced cells, and the ionomycin-induced

276 decrease of Snail levels was only partially attenuated in both CypB and CypA-silenced cells.

277 These changes occurred independently of ionomycin-induced ERK activation. Taken together

278 these results suggest that CypB could be regulating Slug levels through ER calcium-related

279 events.

\section{CypB depletion ameliorates inflammation and fibrosis after UUO}

To expand on the results performed in vitro and to assess the potential contribution of CypB in the development of fibrosis, global CypB-KO mice and wild-type (wt) littermates (Fig. $6 \mathrm{~A})$ were subjected to unilateral ureteral obstruction (UUO) of the left kidney for one-week period to mimic the pathological conditions underlying early events in renal fibrosis (Fig. 6B). Controls corresponded to contralateral (non-ligated) right kidneys from the same mice. Our results show that while no apparent differences were observed between the non-ligated kidneys from wt and CypB KO mice regarding overall kidney morphology $(\mathrm{HE})$, inflammation ( $\mathrm{F} 4 / 80)$ and collagen deposition (MT), we found that obstructed kidneys from CypB KO mice were protected from the tubular distension, inflammation and fibrosis observed in wt mice under UUO (Fig. 6C). Sham-operated mice were also included and results were the same as those found in nonligated kidneys (not shown). These results suggest that CypB deficiency is associated to reduced UUO-induced kidney fibrosis.

Since UUO induces a robust inflammatory response we compared the levels of proinflammatory cytokines present in obstructed kidneys of wt and CypB KO mice (Fig. 6D). We found that in wt mice the levels of tumor necrosis factor $\alpha$ (TNF- $\alpha$ ), macrophage chemoattracting protein 1 (MCP-1) and the pan-macrophage marker CD68 were strongly up-regulated in the obstructed kidneys in comparison to the right contralateral kidneys. This increase was significantly reduced in the kidneys of CypB KO mice. In non-obstructed kidneys, there were no 
300

301

302

303

304

305

306

307

308

309

310

311

312

differences in the expression of these inflammatory markers between wt and CypB KO mice. In accordance with the protein levels shown in Figure 6A, CypB mRNA levels were almost undetectable in CypB KO mice and were not affected by the UUO (Figure 6D). We also observed that the genetic deletion of CypB prevented the down-regulation of CypA induced by UUO.

To further investigate the effects of CypB deletion in kidney fibrosis we analyzed the levels of fibrosis and EMT related genes. In obstructed kidneys of wt mice, we observed a potent and significant increase in the expression levels of ECM components fibronectin and collagen-la and the metalloproteinase MMP-9, as well as of TGF $\beta$. We also observed a significant reduction of E-cadherin and CD147 mRNA levels in comparison with those found in the contralateral kidneys (Fig. 6E). Our results show that genetic deletion of CypB significantly prevented the UUO-induced increase of fibronectin and MMP-9 and the decrease of E-cadherin and CD-147 observed in wt mice. However, we didn't observe significant differences in TGF $\beta$ levels between wt and CypB KO mice that could explain the aforementioned protective effects of CypB knockdown. To gain further insights into the mechanism of action of CypB, we next investigated the levels of the TGF $\beta$ downstream mediators Snail and Slug, and the levels of the antifibrotic factors SMAD7, BMP7 and BMP6, which counteract the TGF $\beta$ pathway. Kidney obstruction increased the levels of Slug, Snail, SMAD7 and BMP6 while reduced those of BMP7 (Fig. 6E). CypB knockdown reduced Slug levels both in contralateral and obstructed kidneys, while increased those of Snail only after UUO. CypB KO mice also show a reduced decrease and an augmented increase in BMP7 and BMP-6, respectively, after UUO. Finally, we also observed that SMAD7 levels were upregulated in non-ligated kidneys of CypB KO mice.

These results, together with those found in HK-2 cells, indicate the association of CypB deficiency and a lower fibrotic and inflammatory response after UUO and suggest a potential role of CypB on EMT processes. 
It is increasingly accepted that after sustained kidney injury, tubular epithelial cells undergo a partial EMT or type 2 EMT, which contributes to kidney fibrosis. This process appears to be plastic, where cells are not engaged in an unidirectional process or locked into one differentiated state, but eventually transit back to the epithelial phenotype (43). Accordingly, the identification of factors regulating this epithelial plasticity could improve the understanding of kidney fibrosis. In the present work, we demonstrate for the first time that CypB is a critical regulator of tubule epithelial cell plasticity on the basis that: i) CypB silencing caused epithelial differentiation in the proximal tubule-derived cell line HK-2, ii) CypB silencing prevented TGF $\beta$ induced EMT in HK-2 cells, and iii) Global CypB knockdown is associated to a reduced UUOinduced kidney fibrosis. Interestingly, silencing of CypA in HK-2 cells exerted almost diametrically opposite effects on epithelial cells than CypB silencing. All these effects most likely result from CypB and CypA regulation of the transcriptional repressor Slug and Snail. folding and that they are also involved in multiple cellular processes such as cell division, protein trafficking, cell signaling, transcriptional regulation and stress tolerance among others (44). Results presented in this study show that in a global CypB knockdown the levels of inflammation and fibrosis mediators were reduced and that the loss of E-cadherin in mouse obstructed kidneys was prevented. Since a pro-inflammatory role of extracellular CypB has been described (45), it could not be discarded that the protective effects of CypB knockdown on kidney fibrosis were secondary to a reduction in inflammation. Our results in HK-2 cells not only support a direct effect of CypB silencing on promoting epithelial phenotype but also in reducing inflammation through NFKB inhibition. Altogether, our results suggest that the gain of epithelial markers observed in CypB silenced proximal tubule cells might resemble the mesenchymal to epithelial (MET) transition undergone by surviving tubule cells upon injury, thus promoting the functional and histological features that would return the kidney to normal function. Accordingly, it could be expected that CypB overexpression recapitulated events driving to a more dedifferentiated phenotype. Actually, it has been described that CypB overexpression is related to malignant progression in several types of tumors including breast cancer, hepatocellular carcinoma, gastric tumors and malignant gliomas (46-49). Contrarily to the effects caused by 
CypB silencing, knockdown of CypA induced a more undifferentiated phenotype regarding the molecular and functional epithelial markers studied. These results are in agreement with previous studies showing loss of cell-cell contacts and increased fibronectin expression after CypA-silencing of human renal epithelial cells from nephrectomy specimens (50). Considering the above phenotypical consequences of CypA silencing in cultured cells, the reduction in CypA levels observed in mouse kidneys after ureteral obstruction could be relevant for the development of fibrosis. In this sense, the increased levels of CypA in injured kidneys of СypB KO mice may respond to a physiological compensatory mechanism. In summary, results obtained in both, СypB KO mice and in cultured proximal tubule cells demonstrate a role for CypA and СypB in epithelial plasticity and homeostasis; while CypB would foster a more dedifferentiated state, CypA would preserve the epithelial phenotype.

These striking opposite actions of CypB and CypA silencing on the HK-2 epithelial phenotype revealed, for the first time, a differential regulation of Snail and Slug transcriptional repressors by these cyclophilins. Specifically, CypA-silenced cells showed upregulated levels of both Snail and Slug while CypB-silencing strongly downregulated Slug and upregulated Snail; thereby indicating that while CypB represses Snail but is required for Slug expression, CypA acts as a repressor of both Slug and Snail proteins. Moreover, we can conclude that, at least in CypB silenced cells, loss of Slug drives the epithelial cell phenotype in spite of increased Snail levels. In this sense, and despite their high homology in their DNA binding and SNAG domains, Snail and Slug present common and nonequivalent functions in epithelial promoter repression, DNA binding and EMT-inducing ability (51). This predominant role of Slug is also particularly relevant since Snail has been getting most of the attention and considered as a sufficient and necessary factor to induce EMT and fibrosis in mouse kidney (11). fibrosis. In cultured proximal tubular cells, including HK-2, treatment with TGF $\beta$ is enough to induce Slug and Snail and trigger EMT. Consistent with our results in untreated cells, CypB silencing prevented TGF $\beta$-induced Slug expression and its profibrotic effects, while CypA silencing enhanced them. TGF $\beta$ regulates renal fibrosis positively by receptor regulated RSmads (Smad2/3), but negatively by inhibitory I-Smads (Smad6/7) which are transcriptionally induced by TGF $\beta$ establishing an important negative feedback loop. In this sense, blockade of 
TGF $\beta$ signaling by Smad7 gene therapy is known to prevent experimental renal fibrosis (52). Interestingly, it is worth noticing that, from the gene panel analyzed, Smad7 and Slug were the only genes differentially expressed in non-ligated kidneys of CypB KO in comparison with wt mice; where Smad7 mRNA overexpression was associated with Slug down-regulation. In agreement with that, we found that in TGF $\beta$ treaded HK-2 cells, Smad7 and also Smad6 expression was upregulated in CypB-silenced cells. I-Smads antagonize TGF- $\beta$ signaling through multiple negative feedback mechanisms that include: i) formation of Smad7 stable complexes with activated type I TGF $\beta$ receptor ALK5/TRRI which blocks the phosphorylation of R-Smads and subsequent nuclear translocation of R-Smad/Smad4 heterocomplexes; ii) by recruiting ubiquitin E3 ligases, such as Smurf1/2, resulting in the ubiquitination and degradation of $T \beta R I$ and iii) in the nucleus, by interfering with the formation of the functional RSmad/Smad4-DNA complex on target gene promoters (53). On this basis and considering that CypA and CypB silencing modulate Slug and Snail levels upon TGF $\beta$ treatment without changes in either Smad2/3 phosphorylation or nuclear translocation, our results would suggest a direct inhibitory effect of Smad7 over Slug promoter in CypB-silenced cells. This mechanism seems to be specific for Slug promoter, since it is not affecting Snail expression. Besides TGF $\beta$, Smad7 expression is also regulated by BMPs. BMPs further counteract TGF $\beta$ pathway through other mechanisms such as control of Snon expression, which represses TGF $\beta$ signaling by inactivating Smad transcriptional complexes. Considering that levels of BMP7 and 6 in CypB KO mice and BMP-2 and Snon in CypB-silenced HK-2 cells were upregulated, and that CypB knockdown had no significant effect on UUO-induced TGF $\beta$ levels, our results suggest that lack of CypB would impact Slug expression by counteracting, rather than hampering, TGF $\beta$ signaling. different mechanisms. Thus, while the PPlase activity of CypA is required to keep both Slug and Snail downregulated, the presence of CypB signal peptide, but not its PPlase activity, is mandatory to allow slug expression. These results fit with a previous report showing that CypA, through its PPlase activity, participates in epithelial differentiation of kidney intercalated cells by mediating matrix assembly of the extracellular matrix protein hensin (54). The involvement of the PPlase activity of CypA in the maintenance of epithelial phenotype was also supported by the 
415 fact that, although cyclosporine-A (CsA) inhibits the PPlase activity of both CypB and CypA,

416 silencing of CypA, rather than that of CypB, mimics the CsA-induced phenotypic changes

417 previously described on proximal tubular cells (13), pointing to CypA as the main target of CsA

418 effects. Regarding CypB, the involvement of its signal peptide on slug expression might either require progression through the secretory pathway ultimately leading to secretion, or the need for CypB localization within the ER. Considering the aforementioned extracellular role of cyclophilins, the existence of a cyclophilin autocrine signaling loop affecting slug expression could not be discarded. Nevertheless, our results argue against an extracellular role of CypB in modulating slug since: i) no differences in either СypB or СypA secretion were observed after TGF $\beta$ treatment; ii) silencing of CD147, the only known receptor for extracellular cyclophilins, did not prevent but rather increase slug expression; and iii) exogenously added recombinant CypB was unable to restore slug levels. It is worth mentioning that the effects of CD147 silencing on Slug expression resembled those of CypA silencing, suggesting that, at least for CypA, an extracellular loop could be involved in CypA regulation of Slug and Snail. In accordance with this, it has been previously described that CypA acts as a survival-enhancing autocrine factor in mouse ESC cultures (55). Moreover, it has been reported that extracellular CypA activates the SMAD pathway and promotes inflammation in biliary atresia and that targeting this extracellular CypA ameliorates disease progression (56). expression by means of its non-catalytic chaperone role within the ER. From the initial discovery of CypB interaction with CAML (calcium-signal modulated cyclophilin ligand) (57), novel CypB partners have been identified, including the sodium-potassium ATPase (58) and the human TRPV6 calcium channel protein (59) among others. In addition, CypB participates in macromolecular chaperones complexes in the ER lumen together with the ERp72, CRT, CNX, BiP (GRP78) and GRP94 $(60,61)$, and the lectin chaperones calnexin (CNX) and calreticulin (CRT) (39). The latest are of special interest because their involvement in calcium signaling and to previous reports indicating that CRT was required for TGF $\beta$-induced EMT $(41,42,62-64)$. The biological and functional relevance of CypB interaction with CRT and CNX has not been still elucidated. Our results show that in HK-2 cells, CypB preferentially interacts with CRT since no interaction with CNX was observed under control conditions. Interestingly, CypB-CRT 
interaction was increased when the PPlase activity of CypB was muted, and was totally prevented when CypB was not present in the ER, closely paralleling that observed in Slug expression and therefore suggesting a potential role of CypB-CRT interaction in Slug regulation. According to the literature, and as we observed in CypB-silenced cells, CRT seem to be preferentially acting over Slug, since CRT over expression in kidney epithelial MDCK cells (40) or HK-2 cells (42), resulted in Slug upregulation without apparent (40), or much more reduced (42), changes in Snail levels. Moreover, as occurred in CypB silenced HK-2 cells, CRT depletion does not impact canonical TGF $\beta$ signaling as TGF $\beta$ was still able to stimulate Smad activity in CRT -/- MEFs (63). On the other hand, interaction of CypB with CNX was only detected when CypB has its PPlase activity mutated or when CypB was unable to enter the ER. CypB interacts with CRT and CNX through their P-domain, and complex formation is not affected by CsA, confirming the functional independence of the P-domain binding and PPlase activity (65). We hypothesize that, since CypB interaction with other partners might require intact PPlase residues, CRT/CNX interaction with $\triangle P P I C y p B$ would benefit from a larger pool of unbound CypB. Nevertheless, interaction of CNX with $\triangle E R$ CypB remains to be explained considering that $\mathrm{CNX}$ is an integral ER transmembrane protein and that the $\mathrm{p}$-domain containing CypB interaction sites faces the lumen of the ER. Considering our results and previous data on the literature, it is entirely plausible that СypB could be modulating Slug expression through its interaction with CRT.

It has been described that CRT mediates TGF $\beta$-dependent transcriptional responses through its role as a calcium signaling regulator, rather than through its chaperone/UPR function (64). In this sense, cells lacking CRT have impaired calcium release from the ER (64), suggesting that calcium release could be a key factor in Slug modulation. However, our results suggest that an increase in cytosolic calcium levels per se is not determinant in slug modulation, since treatment with ionomycin or thapsigargin $(\mathrm{Tg})$, which both ultimately lead to increased cytosolic calcium, exerted opposite effects on Slug levels, by either increasing or decreasing them, respectively. Thus, it is likely that a more specific calcium event within the ER underlies

472 Slug modulation by СурВ. Similar results were obtained by Zimmerman et al. (63), showing that 473 TGF $\beta$ treatment in the presence of ionomycin, despite an increase in cytoplasmic calcium, was still unable to increase ECM transcript in CRT-deficient cells. Results from these authors 
suggested that, although CRT-mediated calcium regulation is a critical factor for TGF $\beta$-induced

476 ECM stimulation, calcium itself is not sufficient, and other CRT-dependent factors should be

477 involved. In a similar manner, we observed that CypB silencing prevented the increase in Slug

478 levels after lonomycin treatment. Ionomycin is $\mathrm{a} \mathrm{Ca}^{2+}$ ionophore, while thapsigargin irreversible

479 inhibits the SERCA pump, abrogating the reuptake of cytosolic calcium into the ER, and thus

480 causing depletion of $\mathrm{Ca}^{2+}$ within the ER. Since both thapsigargin-induced SERCA inhibition and

481 CypB silencing had the same effects on Slug expression, and it has been described that CRT regulates SERCA activity (66), we hypothesize that the lack of Slug expression on CypBsilenced cells could result from impaired CRT modulation of SERCA activity. In addition, it has been described that CypB overexpression protects against thapsigargin-induced cell death, attenuating calcium release from the ER (67). Taken together, our results indicate that CypB regulation of Slug could be related to the indirect action of CypB on ER calcium stores through interaction with the major calcium binding chaperone CRT. tubular cell phenotype in differentiation and EMT processes, most likely through regulation of the expression of the transcriptional repressors Slug and Snail. Our results also reconsider the functional relevance between both repressors, placing Slug as a key regulator of EMT in the fibrotic kidney. As modulators of EMT, targeting CypA or CypB could have a great impact not only on the overall outcome of kidney fibrosis but also in other processes where cell-cell contacts are critical, such as in cancer. Moreover, we also propose a crucial role of CypB in regulating the inflammatory response that precedes kidney fibrosis, establishing $\mathrm{CypB}$ as an important link between inflammation and fibrogenesis. Finally, development of drugs specifically targeting CypB could have a therapeutic benefit to reduce TGF $\beta$ effects and ameliorate kidney fibrosis. 


\section{Unilateral ureteral obstruction (UUO) procedure} midline abdominal incision, the left kidney was exposed by retraction of the intestines using a self-retaining microdissection retractor and the left ureter was carefully dissected from surrounding tissue. The ureter was then doubly ligated at the midpoint between the kidney and the bladder using sterile 6-0 silk suture. The surgical incision was then closed and the mouse was allowed to recover from anesthesia; postoperative analgesia (buprenorphine, $0.1 \mathrm{mg} / \mathrm{kg}$, SQ) was administered. Sham-operated control mice underwent a similar surgical procedure without ligation of the ureter.

\section{RNA extraction and RT-PCR}

Total RNA was isolated using TRIzol® Reagent (\#15596-026, Ambion, Life Technologies) according to manufacturer's instructions. Reverse transcription was performed from $2 \mu \mathrm{g}$ of total RNA with kit High-Capacity RNA-to-cDNA ${ }^{\text {TM }}$ Kit (Applied Biosystems, \#4387406). Quantitative RT-PCR was carried out on an ABI PRISM 7900 Sequence Detection System (Applied Biosystems) using pre-designed FAM-labeled TaqMan probes (Applied Biosystems). All probes used in this work are listed in Supplemental Material. Analysis of relative gene expression data was performed using the $2^{-(\Delta \Delta C t)}$ method after normalizing to TBP.

\section{Cell culture} attributes of normal adult human proximal tubular epithelium (69), were cultured in medium A

524 (DMEM:Ham's F12 (1:1, v/v), 20 mM HEPES, 2mM L-glutamine, 12.5 mM D-glucose, $60 \mathrm{nM}$ 525 sodium selenite, $5 \mu \mathrm{g} / \mathrm{ml}$ transferrin, $50 \mathrm{nM}$ dexamethasone, $100 \mathrm{U} / \mathrm{ml}$ penicillin and $100 \mu \mathrm{g} / \mathrm{ml}$ 
526

527

528

529

530

531

532

533

534

535

536

537

538

539

540

541

542

543

544

545

546

547

548

549

550

551

552

streptomycin) supplemented with $2 \%$ fetal bovine serum (FBS), $5 \mu \mathrm{g} / \mathrm{ml}$ insulin, $10 \mathrm{ng} / \mathrm{ml}$ epidermal growth factor (EGF) and $1 \mathrm{nM}$ triiodothyronine, at $37^{\circ} \mathrm{C}$ in a $95: 5$ air: $\mathrm{CO}_{2}$ watersaturated atmosphere. For all experiments, cells were seeded at $0.1 \times 10^{6}$ cells $/ \mathrm{ml}$. For TGF $\beta$ treatments, cells were starved in medium A supplemented with only $0.1 \%$ FBS and without insulin, EGF or triiodothyronine (starvation medium). After 16 hours starvation, medium was removed and cells were treated for the indicated times with fresh starvation medium containing $1.5 \mathrm{ng} / \mathrm{ml}$ TGF $\beta$ (\#100-B-001, R\&D Systems). When indicated, cells were treated with the indicated doses of human TGF $\beta$ (\#100-B-001, R\&D Systems, Minneapolis, MN, USA), MG-132 (\#BML-PI102, Enzo Life Sciences, Farmingdale, NY, USA), Cyclosporine-A (\#239835, Calbiochem, San Diego, CA, USA), Brefeldin-A (\#B6542, Sigma-Aldrich, St. Louis, MO, USA), human CypB (\#NBC1-18424, Novus Biologicals, Littleton, CO, USA), Ionomycin (\#I0634, Sigma-Aldrich) Thapsigargin (\#586005, Calbiochem).

\section{Gene silencing}

CypA, CypB and CD147 silencing was performed as described in (58). For CypA and CypB silencing, shRNA-containing lentiviral particles were generated by co-transfecting HEK293T cells with second generation vectors including the transfer vector pAPM, carrying the shRNA and puromycin resistance, the HIV-1 packaging plasmid psPAX2, and a VSVg expression plasmid (pMD2.G) (complete information about these vectors is available at (70)). For CD147 silencing, a MISSION® TRC shRNA transfer vector (TRCN0000006732) was cotransfected with the third generation vectors VSVG, RTR2 and PKGPIR, which provide the envelope, packaging and reverse-expressing proteins, respectively. Viral supernatants were then harvested, supplemented and added to HK-2 cells in the presence of polybrene (Hexadimethrin bromide, Sigma-Aldrich). shRNA sequences were indicated in supplemental material.

\section{Co-Immunoprecipitation}



$\mathrm{HCl} \mathrm{pH} \mathrm{7.5,} 150 \mathrm{mM} \mathrm{NaCl}, 2 \mathrm{mM}$ EDTA, $10 \%$ glycerol and $1 \%$ Triton) containing protease and phosphatase inhibitors, and $750 \mu \mathrm{g}$ of cell extracts were incubated with $40 \mu \mathrm{l}$ of anti-HA Affinity hour at $37 \stackrel{\circ}{\mathrm{C}}$.

\section{Western blot}

Cells were lysed with RIPA buffer supplemented with protease inhibitor cocktail (SigmaAldrich) and the protein content of cellular extracts was quantified by the BCA assay (Thermo Scientific). Equal amounts of whole cell extract protein were run on SDS PAGE gels and transferred onto PVDF membranes. Membranes were then blocked 1 hour at RT and incubated overnight at $4{ }^{\circ} \mathrm{C}$ with the corresponding antibodies (a complete list of all antibodies used in this work is shown in Supplemental Material). Finally, membranes were developed with the enhanced chemiluminescence method (Millipore) and exposed on hyperfilm. For western blots of extracellular cyclophilins, extracellular media were collected and centrifuged 5 minutes at $1500 \mathrm{~g}$. Thereafter, $200 \mu \mathrm{l}$ of each supernatant was mixed with $50 \mu \mathrm{l}$ of $5 \mathrm{x}$ sample buffer, and 50 $\mu \mathrm{l}$ of the resulting mix loaded on the western blot.

\section{Enzymatic activity assays}

For determination of alkaline phosphatase (AP), dipeptidyl peptidase-4 (DPP4) and - $^{-}$ glutamyltransferase (GGT-2) activities, HK-2 cells were grown for 2, 5, and 10 days, and whole cell extracts were prepared with mannitol buffer (50mM D-mannitol, $2 \mathrm{mM}$ Tris and $0.1 \%$ Triton

$577 \mathrm{X}-100)$ supplemented with protease inhibitor cocktail. AP and DPP4 activities were analyzed as described in (71). For AP activity assay, $50 \mu \mathrm{g}$ of protein in a final volume of $50 \mu \mathrm{l}$ of mannitol 
580 Sigma-Aldrich) for $15 \mathrm{~min}$ at $37^{\circ} \mathrm{C}$ and the absorbance was then measured at $405 \mathrm{~nm}$. For

581 DPP4 activity assay, $50 \mu \mathrm{g}$ of protein in $90 \mu$ l of mannitol buffer were mixed with $10 \mu \mathrm{l}$ of $1.4 \mathrm{M}$

582 glycine- $\mathrm{NaOH}$ pH 8.7 and incubated with $100 \mu \mathrm{l}$ of $1.5 \mathrm{mM}$ glycyl-L-proline-p-nitroanilide for 30

$583 \min$ at $37^{\circ} \mathrm{C}$. Reaction was stopped by adding trichloroacetic acid. Samples were centrifuged

584 and $50 \mu$ l of supernatant were mixed with $50 \mu$ l of cold $0.2 \%$ sodium nitrite and incubated for 10

$585 \min$ at $4{ }^{\circ} \mathrm{C}$. The mixture was then incubated with $50 \mu \mathrm{l}$ of $0.5 \%$ ammonium sulfamate for $2 \mathrm{~min}$,

586 at the end of which $100 \mu \mathrm{l}$ of $0.05 \% \mathrm{n}$-(1-naphthyl)- ethanediamine were added to the mix, which

587 was further incubated in the dark at $37^{\circ} \mathrm{C}$ for additional $30 \mathrm{~min}$. Absorbance was then read at

$588548 \mathrm{~nm}$. Finally, GGT activity was analyzed using a Roche/Hitachi Cobas C system. 50 mg of

589 protein in $100 \mu \mathrm{l}$ of manittol buffer were incubated with L- $\mathrm{Y}$-glutamyl-3-carboxy-4-nitranilide as

$590 \quad Y$-glutamil donor in the presence of glycyl-glycine and the rate of formation of 5-amino-2-

591 nitrobenzoate was measured spectrophotometrically at $405 \mathrm{~nm}$. All these experiments were

592 performed at least three independent times in triplicate.

Vectors and Site-directed mutagenesis

For shRNA rescue experiments, wild-type hCypA and wild-type hCypB were cloned into pDONR vectors (pDONR ${ }^{\text {тM } 221, \# 12536-017, ~ I n v i t r o g e n) ~ u s i n g ~ t h e ~ G a t e w a y ~ c l o n i n g ~ s y s t e m ~}$ (Invitrogen). The QuickChange site-directed mutagenesis kit (Agilent Technologies) was then used to introduce the following silent mutations (c. [151C>A; 153G>A; 156G>T;159C>T; $162 \mathrm{~T}>\mathrm{C} ; 165 \mathrm{~T}>\mathrm{C}]$ ) in the shRNA targeting sequence of hCypB (see Figure $2 \mathrm{~A}$ ). Since shRNA against hCypA was directed to the 3' UTR no further modifications were required. The shRNArescuing vectors were additionally used to introduce mutations altering PPlase activity of CypA (c.161_162delinsGC; p.Arg55Ala) and CypB (c.259_260delinsGC; p.Arg62Ala). To generate the ER location mutant of hCypB (c.4_75del; p.2_25del), restriction sites were introduced at both sides of the fragment to be deleted. Once all the mutations were performed, all inserts were subcloned to the final destination vector (pLenti CMV Hygro DEST 117-1, Addgene), containing hygromycin resistance, by Gateway recombination. All primers used were generated with the confirmed by DNA sequencing. 
HK-2 cells were seeded on microscope cover glasses (Marlenfeld GmbH \& Co.KG) for

6125 days, and, when indicated, starved overnight and treated with $1.5 \mathrm{ng} / \mathrm{ml}$ TGF $\beta$ for $24 \mathrm{~h}$. Slides were then washed twice in PBS and fixed in 4\% paraformaldehyde for $1 \mathrm{~h}$. Aldehyde groups were then blocked with $50 \mathrm{mM} \mathrm{NH}_{4} \mathrm{Cl}$ for 30 min and cells were permeabilized with $0.1 \%$ triton X-100 for $10 \mathrm{~min}$. Prior to addition of primary antibodies, unspecific binding sites were blocked with $5 \%$ BSA in PBS for 30 min. Slides were then incubated overnight at $4{ }^{\circ} \mathrm{C}$ with a $1: 100$ dilution of primary antibodies and subsequently labeled with secondary fluorescent antibodies (1:200 dilution; see Supplemental Material for references) and Hoechst $33342(\mathrm{H} 1399$, Invitrogen) for nuclear staining 1 hour at room temperature. Fluorescence labeling was visualized in a confocal spectral FV 1000 Olympus microscope. (CFSE) staining. Cells were tripsinized, washed with PBS, and incubated with CFSE (Invitrogen) at $2.5 \mu \mathrm{M}$ final concentration for $10 \mathrm{~min}$ at $37^{\circ} \mathrm{C}$ in the dark in a cell culture incubator. An aliquot of cells was left unlabeled to set background fluorescence. CFSE was then quenched by washing cells twice with complete medium, and a portion of cells was taken to measure fluorescence at the beginning of the experiment. The rest of labeled cells were plated on six-well plates and incubated at $37^{\circ} \mathrm{C}$ in $5 \% \mathrm{CO}_{2}$ for 5 days. Flow cytometric analysis was performed on a FACScalibur (Becton Dickinson) flow cytometer and analyzed with Cell Quest software (Becton Dickenson). Cell proliferation was expressed as the ratio FInd/MFI, where MFI was the median fluorescence intensity of all viable cells at collection and Find the peak fluorescence intensity of the viable non-divided cells. 
For Transepithelial electrical resistance (TEER) and Fluorescein isothiocyanate-dextran

637 (FITC-Dextran) permeability experiments cells were seeded on 24-well transwell plates with 0.4 $638 \mu \mathrm{m}$ pore polyester membrane inserts (HTS transwell-24 PET; \#CLS3379-2EA, Sigma-Aldrich 639 Saint Louis, Missouri, USA) and measurements were performed 5 days after seeding the cells. 640 TEER was measured using an epithelial voltmeter (Millicell-ERS, Millipore, Billerica, MA, USA) with STX100C electrode (for 24 well format) (World Precision Instruments, Sarasota, FL, USA) according to manufacturer's instructions. For permeability assay, 40 kDa FITC-Dextran (SigmaAldrich) in a final concentration of $100 \mu \mathrm{g} / \mathrm{ml}$ was added to the apical compartment of the cells. 180 minutes after adding FITC-Dextran, 200 ul samples were collected from the basolateral compartment and absorbance was measured at $485 \mathrm{~nm}$ of excitation and $528 \mathrm{~nm}$ of emission with a microplate reader (Spectramax Gemini, Molecular Devices, Sunnyvale, CA, USA). Experiments were performed in triplicate with 8 independent samples per group. plate and incubated for $24 \mathrm{~h}$. Cells were then cotransfected with $500 \mathrm{ng} /$ well of a DNA mix containing reporter plasmid NFKB (Firefly luciferase gene with NFKB promoter) and reporter plasmid RLTK (Renilla luciferase gene with thymidine kinase promoter) in a 5:1 ratio, plus either negative control plasmid (pCMV-HA) or positive control plasmid (p65 subunit of NFKB). Transfection was performed using Lipofectamine 3000 transfection kit (\#L3000-001; Thermo Fisher Scientific, Waltham, MA USA) according to the manufacturer's instructions. Cells were lysed and luciferase assay was performed using a Dual-Luciferase® Reporter Assay System (\#E1910, Promega, Fitchburg, WI, USA). Transfection efficiency was normalized by the value of cotransfected Renilla luciferase.

\section{Cell Adhesion assay}


664 were then trypsinized and washed twice with culture medium to eliminate trypsin. Subsequently,

665 cells were counted and $2 \times 10^{4}$ cells/well were seeded onto two duplicated 96-well plates. Cells

666 were then allowed to adhere at $37^{\circ} \mathrm{C}$ for 30 minutes. After the incubation period, unattached

667 cells from one of the plates were removed by washing twice with PBS. The amount of the

668 remaining attached cells (from the washed plate) and the total of cells (from the unwashed

669 plate) was determined using the XTT assay. For each treatment condition, results were

670 expressed as the ratio of XTT values from washed and unwashed plate.

672 Statistics

Results were expressed as the mean \pm standard error of the mean (SEM). Experiments performed in mice were analyzed by two-way analysis of variance (ANOVA) followed by Tukey multiple comparison test. For the remaining experiments, Student's t-test was used for statistical analysis. A P value of less than 0.05 was considered to indicate statistically-significant

677 differences. Statistical analyses were made with commercially available software (GraphPad

678 Prism, version 6.00 for Windows, GraphPad Software, La Jolla California USA).

\section{Study approval}




\section{Author Contributions}

ES and AM conceived and designed the research studies. ES, MD, AR, AC, KA, MS

685 and JG conducted the experiments. RB provided the CypB KO mice. ES, AM, RB and DB analyzed the data. ES, AM, DB, KA and RB draft the manuscript. All authors read and approved the final manuscript.

688

\section{Acknowledgements}

690

Eduard Sarró and Mónica Durán were supported by the generous contribution of Asdent

691 Patients Association. This work was supported in part by grants from Ministerio de Ciencia e

692 Innovación (SAF2011-2950 and SAF2014-59945-R to A. Meseguer), the Fundación Senefro (SEN to A. Meseguer), Instituto de Salud CarlosIII (PIE13/00027), and Red de Investigación 694 Renal REDinREN (12/0021/0013). Karl A. Nath is supported by NIH DK 47060. Meseguer's 695 research group holds the Quality Mention from the Generalitat de Catalunya (2014 SGR). We 696 want to thank Dr. Santiago Lamas and Dr. José Miguel López-Novoa for critical reading of this manuscript. 


\section{References}

1. Declèves AE, Sharma K. Novel targets of antifibrotic and anti-inflammatory treatment in CKD [Internet]. Nat. Rev. Nephrol. 2014;10(5):257-267.

2. Lovisa S et al. Epithelial to Mesenchymal Transition induces cell cycle arrest and parenchymal damage in renal fibrosis. Nat. Med. 2016;21(9):998-1009.

3. Grgic I et al. Targeted proximal tubule injury triggers interstitial fibrosis and glomerulosclerosis [Internet]. Kidney Int. 2012;82(2):172-183.

4. Meng X, Nikolic-Paterson DJ, Lan HY. TGF- $\beta$ : the master regulator of fibrosis [Internet]. Nat. Rev. Nephrol. 2016;12(6):325-338.

5. Bonventre J V., Zuk A. Ischemic acute renal failure: An inflammatory disease? In: Kidney International. 2004:
6. Torres IB, Moreso F, Sarró E, Meseguer A, Serón D. The Interplay between Inflammation and Fibrosis in Kidney Transplantation2014;2014.

7. Iwano $\mathrm{M}$ et al. Evidence that fibroblasts derive from epithelium during tissue fibrosis. J. Clin. Invest. 2002;110(3):341-350.

8. Peinado H, Olmeda D, Cano A. Snail, ZEB and bHLH factors in tumour progression: An alliance against the epithelial phenotype?. Nat. Rev. Cancer 2007;7(6):415-428.

9. Bolos V et al. The transcription factor Slug represses E-cadherin expression and induces epithelial to mesenchymal transitions: a comparison with Snail and E47 repressors [Internet]. J. Cell Sci. 2016;129(6):1283-1283.

10. Boutet A, Esteban MA, Maxwell PH, Nieto MA. Reactivation of Snail genes in renal fibrosis and carcinomas: A process of reversed embryogenesis?. Cell Cycle 2007;6(6):638-642.

11. Grande MT et al. Snail1-induced partial epithelial-to-mesenchymal transition drives renal fibrosis in mice and can be targeted to reverse established disease. Nat. Med. 2015;21(9):989-997.

12. Bennett WM, Demattos A, Meyer MM, Andoh T, Barry JM. Chronic cyclosporine nephropathy: The Achilles' heel of immunosuppressive therapy. Kidney Int. 1996;50(4):1089-1100.

13. Slattery C, Campbell E, McMorrow T, Ryan MP. Cyclosporine A-induced renal fibrosis: a role for epithelial-mesenchymal transition. [Internet]. Am. J. Pathol. 2005;167(2):395-407.

14. González-Guerrero C et al. TLR4-mediated inflammation is a key pathogenic event leading to kidney damage and fibrosis in cyclosporine nephrotoxicity. Arch. Toxicol. 2017;91(4):1925-1939.

15. Handschumacher RE, Harding MW, Rice J, Drugge RJ, Speicher DW. Cyclophilin: 
a specific cytosolic binding protein for cyclosporin A. [Internet]. Science 1984;226(4674):544-7.

16. Göthel SF, Marahiel MA. Peptidyl-prolyl cis-trams isomerases, a superfamily of ubiquitous folding catalysts. Cell. Mol. Life Sci. 1999;55(3):423-436.

17. Wang P, Heitman J. The cyclophilins. [Internet]. Genome Biol. 2005;6(7):226.

18. Bernasconi R et al. Cyclosporine A-Sensitive, Cyclophilin B-Dependent endoplasmic reticulum-associated degradation. PLoS One 2010;5(9):1-7.

19. Price ER et al. Cyclophilin B trafficking through the secretory pathway is altered by binding of cyclosporin A.. Proc. Natl. Acad. Sci. U. S. A. 1994;91(9):3931-3935.

20. Tegeder I et al. Elevated serum cyclophilin levels in patients with severe sepsis [Internet]. J Clin Immunol 1997;17(5):380-386.

21. Billich A, Winkler G, Aschauer H, Rot A, Peichl P. Presence of cyclophilin A in synovial fluids of patients with rheumatoid arthritis [Internet]. J Exp Med 1997;185(5):975-980.

22. De Ceuninck F, Allain F, Caliez A, Spik G, Vanhoutte PM. High binding capacity of cyclophilin B to chondrocyte heparan sulfate proteoglycans and its release from the cell surface by matrix metalloproteinases: Possible role as a proinflammatory mediator in arthritis. Arthritis Rheum. 2003;48(8):2197-2206.

23. Xu Q, Leiva MC, Fischkoff SA, Handschumacher RE, Lyttle CR. Leukocyte chemotactic activity of cyclophilin. J. Biol. Chem. 1992;267(17):11968-11971.

24. Allain F et al. Interaction with glycosaminoglycans is required for cyclophilin B to trigger integrin-mediated adhesion of peripheral blood T lymphocytes to extracellular matrix [Internet]. Proc. Natl. Acad. Sci. 2002;99(5):2714-2719.

25. Yurchenko V, Constant S, Bukrinsky M. Dealing with the family: CD147 interactions with cyclophilins. Immunology 2006;117(3):301-309.

26. Cebrián C et al. Kidney Androgen-regulated Protein Interacts with Cyclophilin B and Reduces Cyclosporine A-mediated Toxicity in Proximal Tubule Cells. J. Biol. Chem. 2001;276(31):29410-29419.

27. Tornavaca $\mathrm{O}$ et al. KAP degradation by calpain is associated with $\mathrm{CK} 2$ phosphorylation and provides a novel mechanism for cyclosporine A-induced proximal tubule injury. [Internet]. PLoS One 2011;6(9):e25746.

28. Suñé $\mathrm{G}$ et al. Cyclophilin B interacts with sodium-potassium ATPase and is required for pump activity in proximal tubule cells of the kidney. PLoS One 2010;5(11). doi:10.1371/journal.pone.0013930

29. Liu WF, Nelson CM, Pirone DM, Chen CS. E-cadherin engagement stimulates proliferation via Rac1. J. Cell Biol. 2006;173(3):431-441. 
30. Panchapakesan U. DPP4 Inhibition in Human Kidney Proximal Tubular Cells Renoprotection in Diabetic Nephropathy? [Internet]. J. Diabetes Metab. 2013;S9:1-8.

31. Min HS et al. Dipeptidyl peptidase IV inhibitor protects against renal interstitial fibrosis in a mouse model of ureteral obstruction [Internet]. Lab. Investig. 2014;94(6):598-607.

32. Lamouille S, Xu J, Derynck R. Molecular mechanisms of epithelial-mesenchymal transition.. Natl. Rev. Mol. Cell Biol. 2014;15(3):178-196.

33. Yan X, Liu Z, Chen Y. Regulation of TGF-beta signaling by Smad7.. Acta Biochim. Biophys. Sin. (Shanghai). 2009;41(4):263-272.

34. Meng X, Nikolic-Paterson DJ, Lan HY. Inflammatory processes in renal fibrosis. [Internet]. Nat. Rev. Nephrol. 2014;10(9):493-503.

35. Torres IB, Moreso F, Sarró E, Meseguer A, Serón D. The interplay between inflammation and fibrosis in kidney transplantation. Biomed Res. Int. 2014;2014. doi: $10.1155 / 2014 / 750602$

36. Wu Y et al. Stabilization of snail by NF-kappaB is required for inflammationinduced cell migration and invasion. [Internet]. Cancer Cell 2009;15(5):416-28.

37. Bukrinsky MI. Cyclophilins: Unexpected messengers in intercellular communications. Trends Immunol. 2002;23(7):323-325.

38. Yurchenko V et al. CD147 is a signaling receptor for cyclophilin B.. Biochem. Biophys. Res. Commun. 2001;288(4):786-788.

39. Kozlov $\mathrm{G}$ et al. Structural basis of cyclophilin B binding by the calnexin/calreticulin P-domain. J. Biol. Chem. 2010;285(46):35551-35557.

40. Hayashida $\mathrm{Y}$ et al. Calreticulin represses E-cadherin gene expression in MadinDarby canine kidney cells via slug. J. Biol. Chem. 2006;281(43):32469-32484.

41. Wu Y et al. Calreticulin regulates TGF- $\beta 1$-induced epithelial mesenchymal transition through modulating Smad signaling and calcium signaling [Internet]. Int. J. Biochem. Cell Biol. 2017;90:103-113.

42. Prakoura N, Politis PK, Ihara Y, Michalak M, Charonis AS. Epithelial calreticulin up-regulation promotes profibrotic responses and tubulointerstitial fibrosis development [Internet]. Am. J. Pathol. 2013;183(5):1474-1487.

43. Huang S, Susztak K. Epithelial Plasticity versus EMT in Kidney Fibrosis. Trends Mol. Med. 2016;22(1):4-6.

44. Trivedi DK, Yadav S, Vaid N, Tuteja N. Genome wide analysis of Cyclophilin gene family from rice and Arabidopsis and its comparison with yeast. Plant Signal. Behav. 2012;7(12):1653-1666.

45. Bukrinsky M. Extracellular cyclophilins in health and disease [Internet]. Biochim. 
Biophys. Acta - Gen. Subj. 2015;1850(10):2087-2095.

810

811

812

813

814

815

816

817

818

819

820

821

822

823

824

825

826

827

828

829

830

831

832

833

834

835

836

837

838

839

840

841

842

843

844

46. Fang F, Flegler AJ, Du P, Lin S, Clevenger C V. Expression of cyclophilin B is associated with malignant progression and regulation of genes implicated in the pathogenesis of breast cancer. [Internet]. Am. J. Pathol. 2009;174(1):297-308.

47. Kim Y et al. Role of cyclophilin B in tumorigenesis and cisplatin resistance in hepatocellular carcinoma in humans. Hepatology 2011;54(5):1661-1678.

48. Li T et al. Gastric cancer cell proliferation and survival is enabled by a cyclophilin B/STAT3/MIR-520d-5p signaling feedback loop. Cancer Res. 2017;77(5):1227-1241.

49. Choi JW, Schroeder MA, Sarkaria JN, Bram RJ. Cyclophilin B supports MYC and mutant p53-dependent survival of glioblastoma multiforme cells. Cancer Res. 2014;74(2):484-496.

50. Pallet $\mathrm{N}$ et al. Cyclosporine-induced endoplasmic reticulum stress triggers tubular phenotypic changes and death.. Am. J. Transplant 2008;8(11):2283-2296.

51. Villarejo A, Cortés-Cabrera Á, Molina-Ortíz P, Portillo F, Cano A. Differential role of snail1 and snail2 zinc fingers in E-cadherin repression and epithelial to mesenchymal transition. J. Biol. Chem. 2014;289(2):930-941.

52. Chung ACK et al. Smad7 suppresses renal fibrosis via altering expression of TGF$\beta$ /Smad3-regulated microRNAs [Internet]. Mol. Ther. 2013;21(2):388-398.

53. Yan X, Chen YG. Smad7: not only a regulator, but also a cross-talk mediator of TGF-beta signalling [Internet]. Biochem. J. 2011;434(1):1-10.

54. Peng $\mathrm{H}$ et al. Secreted cyclophilin A, a peptidylprolyl cis-trans isomerase, mediates matrix assembly of Hensin, a protein implicated in epithelial differentiation. J. Biol. Chem. 2009;284(10):6465-6475.

55. Mittal N, Voldman J. Nonmitogenic survival-enhancing autocrine factors including cyclophilin A contribute to density-dependent mouse embryonic stem cell growth. Stem Cell Res. 2011;6(2):168-176.

56. Iordanskaia $\mathrm{T}$ et al. Targeting extracellular cyclophilins ameliorates disease progression in experimental biliary atresia. [Internet]. Mol. Med. 2015;21(1):657 - 664 .

57. Bram RJ, Crabtree GR. Calcium signalling in T cells stimulated by a cyclophilin Bbinding protein [Internet]. Nature 1994;371(6495):355-358.

58. Suñé G et al. Cyclophilin B interacts with sodium-potassium ATPase and is required for pump activity in proximal tubule cells of the kidney [Internet]. PLoS One 2010;5(11):e13930.

59. Stumpf T et al. The human TRPV6 channel protein is associated with cyclophilin B in human placenta. J. Biol. Chem. 2008;283(26):18086-18098.

60. Meunier L, Usherwood Y-K, Chung KT, Hendershot LM. A Subset of Chaperones 
845 and Folding Enzymes Form Multiprotein Complexes in Endoplasmic Reticulum to Bind

846 Nascent Proteins. Mol. Biol. Cell 2002;13(December):4456-4469.

847 61. Jansen $\mathrm{G}$ et al. An Interaction Map of Endoplasmic Reticulum Chaperones and 848 Foldases [Internet]. Mol. Cell. Proteomics 2012;11(9):710-723.

849 62. Karimzadeh F, Opas M. Calreticulin Is Required for TGF- $\beta$-Induced Epithelial-to-

850 Mesenchymal Transition during Cardiogenesis in Mouse Embryonic Stem Cells

851 [Internet]. Stem Cell Reports 2017;8(5):1299-1311.

852 63. Zimmerman KA, Graham L V., Pallero MA, Murphy-Ullrich JE. Calreticulin

853 regulates transforming growth factor- $\beta$-stimulated extracellular matrix production. $J$.

854 Biol. Chem. 2013;288(20):14584-14598.

855 64. Owusu BY, Zimmerman KA, Murphy-ullrich JE. The role of the endoplasmic

856 reticulum protein calreticulin in mediating TGF- $\beta$-stimulated extracellular matrix

857 production in fibrotic disease. J. Cell Commun. Signal. [published online ahead of print:

858 2017]; doi:10.1007/s12079-017-0426-2

859 65. Kozlov $\mathrm{G}$ et al. Structural basis of cyclophilin B binding by the calnexin/calreticulin 860 P-domain. J. Biol. Chem. 2010;285(46):35551-35557.

861 66. Michalak M, Parker JMR, Opas M. Ca2+ signaling and calcium binding chaperones 862 of the endoplasmic reticulum. Cell Calcium 2002;32(5-6):269-278.

863 67. Kim J et al. Overexpressed cyclophilin B suppresses apoptosis associated with ROS 864 and Ca2+ homeostasis after ER stress. [Internet]. J. Cell Sci. 2008;121(Pt 21):3636-48.

865 68. Kie J-H, Kapturczak MH, Traylor A, Agarwal A, Hill-Kapturczak N. Heme

866 oxygenase-1 deficiency promotes epithelial-mesenchymal transition and renal fibrosis..

867 J. Am. Soc. Nephrol. 2008;19(9):1681-1691.

868 69. Ryan MJ et al. HK-2: An immortalized proximal tubule epithelial cell line from 869 normal adult human kidney [Internet]. Kidney Int. 1994;45(1):48-57.

870 70. Pertel T et al. TRIM5 is an innate immune sensor for the retrovirus capsid lattice 871 [Internet]. Nature 2011;472(7343):361-365.

872 71. Mazzolini R et al. Brush border Myosin Ia has tumor suppressor activity in the 873 intestine. Proc. Natl. Acad. Sci. 2012;109:1530-1535.

874 72. Lamouille S, Derynck R. Cell size and invasion in TGF- $\beta$-induced epithelial to 875 mesenchymal transition is regulated by activation of the mTOR pathway. J. Cell Biol. $876 \quad 2007 ; 178(3): 437-451$. 
Figure 1. CypB and CypA silencing differentially affects epithelial phenotype of cultured

PTC. To investigate the potential involvement of cyclophilins in the regulation of the epithelial transfected with shRNA-expressing lentiviral vectors against CypA or CypB, respectively. (B) The expression levels of the epithelial markers E-cadherin, ZO-1, occludin and keratin in HK-2 wild-type cells after 2, 5 and 10 days of culture were analyzed by western blot. The expression levels of the above epithelial markers in CypA and CypB-silenced cells after 5 days of culture were determined by immunoblotting (C) and real time quantitative PCR (qPCR) (D). (E) Immunofluorescence staining of E-cadherin (green) in CypA and CypB-silenced cells cultured for 5 days, showing membrane location of E-cadherin in CypB-silenced cells. Calnexin (red) was used to stain endoplasmatic reticulum. (F) Cell proliferation in control and CypA and CypBsilenced cells was measured by means of carboxyfluorescein succinimidyl ester (CFSE) labeling followed by flow cytometry analysis as indicated in Methods. Values indicate the ratio FInd/MFI, where MFI was the median fluorescence intensity of all viable cells at collection and Find the peak fluorescence intensity of the viable non-divided cells. To assess monolayer integrity after CypA and CypB-silencing, transepithelial electric resistance (TEER) (G) and FITClabeled Dextran permeability $(\mathrm{H})$ were measured in cyclophilin-silenced HK-2 cells cultured for 5 days. Enzymatic activities of alkaline phosphatase (AP) (I), dipeptidyl peptidase-IV (DPP-IV) (J) and gamma-glutamyltransferase (GGT) (K) in CypA and CypB-silenced cells after 2, 5 and 10 days of culture. (L) The expression levels of the transcriptional repressors Snail, Slug, Twist1 and Zeb1 were analyzed by real time quantitative PCR (qPCR). Finally, levels of Slug and Snail in HK-2 wild-type cells after 2, 5 and 10 days of culture (M) or in CypA and CypB-silenced cells at 5 days of culture $(\mathrm{N})$ were analyzed by Western Blot. Student's t-test was used to compare shCon vs shCypA or shCon vs shCypB for each culture time point. * $\mathrm{P}<0.05$. 
908 levels of E-cadherin, occludin, fibronectin, actin, CypA and CypB were analyzed by western

909 blot. (B) CypA- and CypB-silenced HK-2 cells were treated with 1,5 ng/ml TGF $\beta$ for $24 \mathrm{~h}$ and the

910 expression levels of E-cadherin, occludin, fibronectin, actin, CypA and CypB were analyzed by

911 western blot. (C) CypA- and CypB-silenced HK-2 cells were treated with 1,5 ng/ml TGF $\beta$ for 24

$912 \mathrm{~h}$ and cells were then visualized under a light microscope. (D) CypA- and CypB-silenced HK-2

913 cells were treated with $1,5 \mathrm{ng} / \mathrm{ml} \mathrm{TGF} \beta$ for $48 \mathrm{~h}$, trypsinized and seeded again. Cell were

914 allowed to adhere for 30 minutes, after which unattached cells were removed and the amount of

915 the remaining attached cells measured as indicated in methods. (E) HK-2 wt cells were treated

916 with $1,5 \mathrm{ng} / \mathrm{ml}$ TGF $\beta$ for the indicated times and the expression levels of Slug and Snail and the

917 phosphorylation status and expression levels of Smad3 and smad2 were analyzed by western

918 blot. (F) Control, CypA and CypB-silenced HK-2 cells were treated with 1,5 ng/ml TGF $\beta$ for $3 \mathrm{~h}$

919 or $24 \mathrm{~h}$ and the expression levels of slug and snail, and the phosphorylation status and

920 expression levels of Smad3, smad2 and smad4 were analyzed by western blot. Panels on the

921 right show the Slug/actin and Snail/actin ratios, referred to control shRNA-treated cells exposed

922 to TGF $\beta$, and the mRNA levels of Slug and Snail analyzed by qPCR. (G) CypA and CypB-

923 silenced HK-2 cells were treated with $1,5 \mathrm{ng} / \mathrm{ml} \mathrm{TGF} \beta$ for $24 \mathrm{~h}$, with the proteasome inhibitor

924 MG132 $(5 \mu \mathrm{M})$ added to cells for the lasts $16 \mathrm{~h}$ of TGF $\beta$ treatment. Slug levels were analyzed

925 by Western blot. (H) Nuclear translocation of Smad3 and Smad2 in CypA and CypB -silenced

926 cells after treatment or not with $1,5 \mathrm{ng} / \mathrm{ml} \mathrm{TGF} \beta$ for $3 \mathrm{~h}$ was detected by immunofluorescence

927 using antibodies against total Smad3 and Smad2 (green). The nuclei were stained with Hoechst

928 (blue). (I) The expression levels of Smad7, Smad6, Snon and BMP-2 in CypA- and CypB-

929 silenced HK-2 cells treated with $1,5 \mathrm{ng} / \mathrm{ml}$ TGF $\beta$ for $24 \mathrm{~h}$ were analyzed by qPCR using specific

930 proves indicated in supplemental materials. Student's t-test was used to compare shCon vs

931 shCypA or shCon vs shCypB for control or TGF $\beta$ treated cells. ${ }^{*} \mathrm{P}<0.05$.

Figure 3. Slug modulation by CypB is independent of the CD147 receptor and

934 extracellular CypB. Since extracellular CypA and CypB have been proposed as inflammatory mediators, we explored a potential autocrine loop in Cyp-mediated regulation of Slug and Snail. (A) NFKB activity was analyzed by cotransfecting cyclophilin-silenced HK-2 cells with plasmids 
under the thymidine kinase promoter, plus a negative control empty plasmid pCMV-HA (empty) or the p65 subunit of NFKB (p65). NFKB activity was analyzed using a luciferase assay kit. Transfection efficiency was normalized by the value of cotransfected Renilla luciferase. (B) HK2 cells were treated with $1,5 \mathrm{ng} / \mathrm{ml} \mathrm{TGF} \beta$ for the indicated times, and the presence of CypA and

942 CypB in the extracellular medium was analyzed by western blot. Culture medium that has not been in contact with cells was used as a negative control. (C) HK-2 cells were treated with $1 \mu \mathrm{M}$

944 Brefeldin-A (Bf-A) for 30 minutes before addition of either $1,5 \mathrm{ng} / \mathrm{ml}$ TGF $\beta$ or $0,5 \mu \mathrm{M}$ Cyclosporine-A (CsA) for 3 hours. Extracellular media were then collected and analyzed by western blot. (D) HK-2 cells were treated with $1 \mu \mathrm{M}$ Brefeldin-A (Bf-A) for 30 minutes before addition of $1,5 \mathrm{ng} / \mathrm{ml}$ TGF $\beta$ and the expression levels of Slug and Snail and the phosphorylation status and expression levels of ERK1/2 analyzed by western blot. (E) HK-2 cells were stably silenced for CD147 as indicated in Methods, and treated or not with 1,5 ng/ml TGF $\beta$ for 3 hours before analyzing the levels of slug and snail and the the phosphorylation status and expression levels of Smad3 and Smad2. (F) Control and CypB-silenced HK-2 cells were treated with increasing doses of recombinant CypB for $3 \mathrm{~h}$ and the expression of Slug and Snail and the phosphorylation status and expression levels of ERK1/2 analyzed by western blot. Student's ttest was used to compare shCon vs shCypA or shCon vs shCypB for control or p65 transfected cells. ${ }^{*} \mathrm{P}<0.05$.

Figure 4. Slug regulation by СурА and СурB depends on PPlase activity of CypA and ER location of CypB shRNA-resistant wild-type (wt) or shRNA-resistant mutants defective in PPlase activity $(\triangle \mathrm{PPI})$ or, in the case of $\mathrm{CypB}$, its signal peptide $(\Delta \mathrm{ER})$, were cloned into lentiviral expression vectors and reintroduced into CypA or CypB-silenced cells to discard offtargets effects of shRNA and to study the involvement of PPlase activity and ER location. (A) Schematic diagram summarizing the different mutations introduced in СypA and СypB expression vectors. (B) The expression levels of CypA wt and mutant forms reintroduced into CypA-silenced cells were determined by Western Blot. $\varnothing$ corresponds to the empty expression vector. (C and D) Western Blot analysis showing that the increase in Slug and Snail levels observed in CypA-silenced cells is prevented by reintroducing HK-2 CypA wt but not the R55A 
expression levels of CypB wt and mutant forms reintroduced into CypB-silenced cells were also analyzed Western Blot in cell extracts and cell supernatants (SN). $\varnothing$ corresponds to the empty expression vector. $(F)$ Immunofluorescence staining of reintroduced CypB wt and the $\triangle P P I$ and $\triangle \mathrm{ER}$ mutants of CypB. CypB wt and $\triangle \mathrm{PPI}$ colocalized with the ER marker calnexin (CNX) while

972 CypB $\triangle$ ER did not. ( $G$ and $H$ ) Western Blot analysis of the expression levels of Slug and Snail, after reintroduction of CypB wt or $\triangle \mathrm{PPI}$ and $\triangle \mathrm{ER}$ mutants, either in untreated cells (G) or in cells treated with $1,5 \mathrm{ng} / \mathrm{ml}$ TGF $\beta$ for 3 hours. Actin ratios are referred to control shRNA-treated cells for Slug and Snail.

Figure 5. CypB could be modulating Slug levels through calcium regulating elements in

the ER. To explore whether the modulatory effects of CypB on Slug levels could be related to calreticulin (CRT) and calnexin (CNX), we analyzed CRT and CNX subcelular localization and protein interactions in HK-2 silenced cells. (A) Immunofluorescence staining of CRT (ER luminal) and CNX (ER transmembrane) was unaffected by either CypA or CypB silencing. (B) Wild type, PPlase and signal peptide defective mutants of CypB were re-introduced in CypBsilenced cells and immunoprecipitated with HA antibody, followed by immunoblotting with CRT, CNX, HA or CypB. (C) To explore if the changes in slug levels could be related to calcium homeostasis disturbance, HK-2 cells were treated with $5 \mu \mathrm{M}$ thapsigargin or $1 \mu \mathrm{M}$ lonomycin for 3 hours and Slug and Snail levels were analyzed by western blot. (D) HK-2 cells were treated with $5 \mu \mathrm{M}$ thapsigargin or $1 \mu \mathrm{M}$ lonomycin for 30 minutes before addition of $1,5 \mathrm{ng} / \mathrm{ml}$ TGF $\beta$ for 3 hours. (E) Control, CypA and CypB-silenced cells were treated with $1 \mu \mathrm{M}$ lonomycin for 3 hours and Slug and Snail levels and the phosphorylation status and expression levels of ERK $1 / 2$ analyzed by western blot.

Figure 6. СурB depletion ameliorates inflammation and fibrosis after UUO. CypB KO mice were used to assess the involvement of CypB in kidney fibrosis. (A) The expression levels of CypB were analyzed by Western Blot in kidneys from CypB KO (СурВ -/-) mice and control wild type littermates (wt). (B) Scheme depicting the experimental approach: CypB-KO mice and control littermates were subjected to unilateral ureteral obstruction as a model of renal fibrosis. Mice were sacrificed 7 days after obstruction and total RNA was extracted from the right contralateral kidneys (CL) and left obstructed kidneys (UUO). (C) Hematoxylin and eosin (H\&E), 
mouse macrophage marker F4/80, and Masson's trichrome (MT) staining of kidneys sections from contralateral kidneys (CL) and obstructed kidneys (UUO) of wt and CypB KO mice. (D)

1000 CypB, MCP1, CD68, TNFa and CypA mRNA levels detected by qRT-PCR. (E) Fibronectin,

1001 collagen-la, MMP9, TGF $\beta$, E-cadherin, CD147, snail, slug, Smad7, BMP-7, and BMP-6 levels detected by qRT-PCR. Each column shows mean \pm SEM; $n=8$ animals per group. Data were

1003 analyzed by two-way analysis of variance (ANOVA) followed by Tukey multiple comparison test. ns $P \geq 0.05 ;{ }^{*} \mathrm{P}<0.05,{ }^{* *} \mathrm{P}<0.01,{ }^{* * *} \mathrm{P}<0.001 ;{ }^{* * * *} \mathrm{P}<0.0001$.

1005

1006

Figure S1. CypB and CypA silencing differentially affects epithelial phenotype of RPTEC.

1007 To validate the results observed in HK-2 cells, we silenced CypA and CypB in the human

1008 proximal tubule derived cell line RPTEC (\#CRL-4031, ATCC). Western Blot shows the decrease

1009 in CypA and CypB expression in RPTEC cells stably transfected with shRNA-expressing lentiviral vectors against CypA or CypB, respectively. The expression levels of the epithelial markers E-cadherin and occludin and those of the transcriptional repressors Snail and Slug at 5 days of culture were also analyzed by western blot. 


\section{Supplemental Material}

1014

1015 Table S1. Antibodies (in alphabetical order)

\begin{tabular}{|l|l|}
\hline Antibody & Reference \\
\hline Actin & \# A5060; Sigma-Aldrich \\
\hline Calnexin & \# MA3-027, Thermo Fisher \\
\hline Calreticulin & \# 12238; Cell Signaling \\
\hline CD147 & \# 376-820 (UM-8D6); Ancell \\
\hline Cyclophilin A & \# 07-313; Millipore \\
\hline Cyclophilin B & \# PA1-027A; Thermo Scientific \\
\hline E-cadherin & \# 610181; BD Transduction Labs \\
\hline ERK1/2 & \# 06-182; Merck-Millipore \\
\hline ERK1/2 (Thr202/204) phosphate & \# 9101; Cell Signaling \\
\hline Fibronectin & \# F6140; Sigma-Aldrich \\
\hline GAPDH & \# MA5-15738, Thermo Fisher \\
\hline HA & \# 1867423; Roche \\
\hline N-cadherin & \# 610920; BD Transduction Labs \\
\hline Occludin & \# 71-1500; Invitrogen \\
\hline Pan-Cytokeratin (AE1/AE3) & Dako (Agilent) \\
\hline Slug & \# 9585; Cell Signaling \\
\hline Smad2 & \# 5339; Cell Signaling \\
\hline Smad2 (Ser465/467) phosphate & \# 3108; Cell Signaling \\
\hline Smad3 & \# 9523; Cell Signaling \\
\hline Smad3 (Ser423/425) phosphate & \# 9520; Cell Signaling \\
\hline Smad4 & \# Sc-7966; Santa Cruz Biotechnology \\
\hline Snail & \# 3895; Cell Signaling \\
\hline ZO-1 & \# 61-7300; Invitrogen \\
\hline
\end{tabular}


Table S2. Taqman Probes (in alphabetical order)

\begin{tabular}{|c|c|c|c|}
\hline Specie & Gene Symbol & Gene Name & Reference \\
\hline \multirow{12}{*}{ Human } & BMP2 & bone morphogenetic protein 2 & Hs00154192_m1 \\
\hline & $\mathrm{CDH} 1$ & cadherin 1, type 1, E-cadherin (epithelial) & Hs01023894_m1 \\
\hline & $\mathrm{CDH} 2$ & cadherin 2 , type $1, \mathrm{~N}$-cadherin (neuronal) & Hs00983056_m1 \\
\hline & KRT8 & keratin 8 & Hs01670053_m1 \\
\hline & OCLN & occludin & Hs00170162_m1 \\
\hline & SKIL & SKI-like oncogene & Hs01045418_m1 \\
\hline & SMAD6 & SMAD family member 6 & Hs00178579_m1 \\
\hline & SMAD7 & SMAD family member 7 & Hs00998193_m1 \\
\hline & SNAI1 & snail family zinc finger 1 & Hs00195591_m1 \\
\hline & SNAI2 & snail family zinc finger 2 & Hs00950344_m1 \\
\hline & TWIST1 & twist family bHLH transcription factor 1 & Hs00361186_m1 \\
\hline & ZEB1 & zinc finger E-box binding homeobox 1 & Hs00611024_m1 \\
\hline \multirow{16}{*}{ Mouse } & BMP6 & bone morphogenetic protein 6 & Mm01332882_m1 \\
\hline & BMP7 & bone morphogenetic protein 7 & Mm00432102_m1 \\
\hline & BSG & basigin CD147 & Mm0116115_m1 \\
\hline & CCL2 & chemokine ( $\mathrm{C}-\mathrm{C}$ motif) ligand 2 & Mm 00441242_m1 \\
\hline & CD68 & CD68 antigen & Mm 03047340_m1 \\
\hline & $\mathrm{CDH} 1$ & cadherin 1 & Mm 01247357_m1 \\
\hline & COL1A2 & collagen, type I, alpha 2 & Mm00483888_m1 \\
\hline & FN1 & fibronectin & Mm01256744_m1 \\
\hline & MMP9 & matrix metallopeptidase 9 & Mm00442991_m1 \\
\hline & PPIA & peptidylprolyl isomerase $A$ & Mm02342430_g1 \\
\hline & PPIB & peptidylprolyl isomerase $B$ & Mm00478295_m1 \\
\hline & SMAD7 & SMAD family member 7 & Mm00484742_m1 \\
\hline & SNAI1 & snail family zinc finger 1 & Mm00441533_g1 \\
\hline & SNAI2 & snail family zinc finger 2 & Mm00441531_m1 \\
\hline & TGFbeta1 & transforming growth factor, beta 1 & Mm01178820_m1 \\
\hline & TNF & tumor necrosis factor & Mm00443258_m1 \\
\hline
\end{tabular}

1019 
Table S3. shRNA sequences

\begin{tabular}{|l|l|l|}
\hline Gene & Localization & Sequence \\
\hline hCypA & 3'UTR & TGGATTGCAGAGTTAAGTTTA \\
\hline hCypB & CDS 149 to 170 & GCCGGGTGATCTTTGGTCTCTT \\
\hline control non-targeting & 3'UTR & $\begin{array}{l}\text { TCTCGCTTGGGCGAGAGTAAG } \\
\text { GTGTATGATACACTTCCTTCTTCTCGAGAAGAAGGAA }\end{array}$ \\
\hline hCD147 & & \\
\hline
\end{tabular}

1023 
A

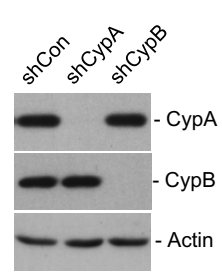

B

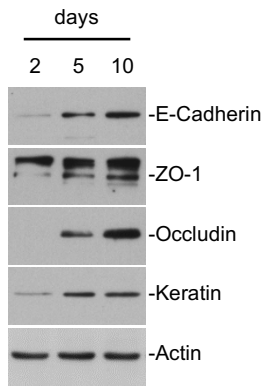

C

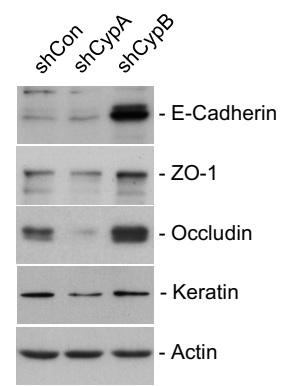

F

shCypB
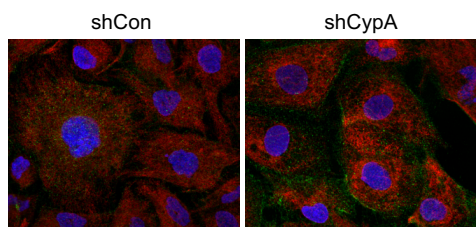

E-cadherin + calnexin

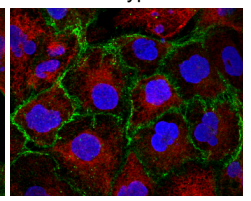

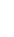

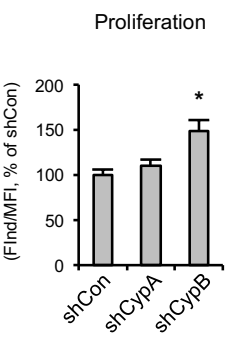

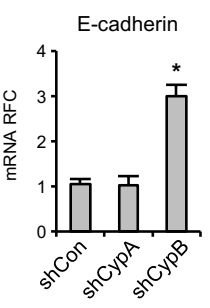
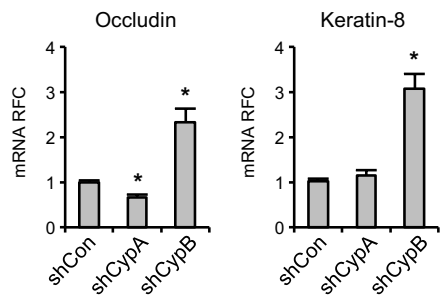

G

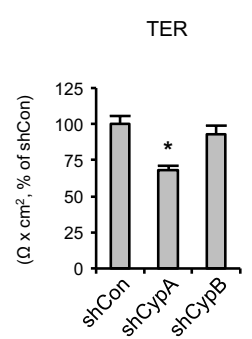

H

$$
\text { FITC-Dextran }
$$

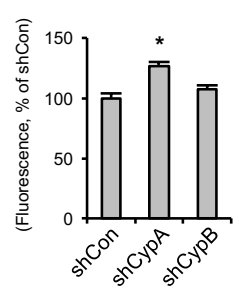

I

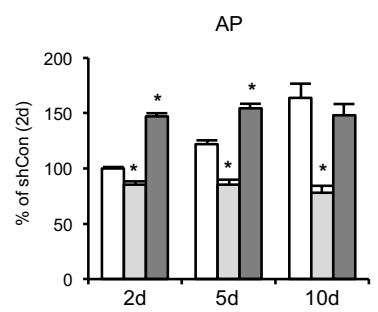

J

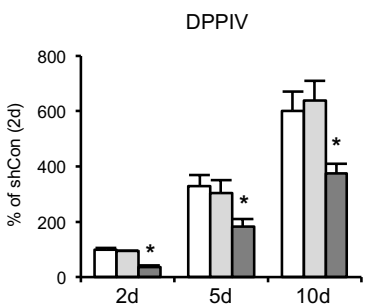

K

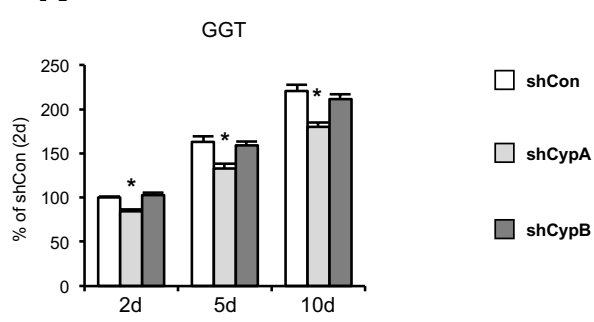

L

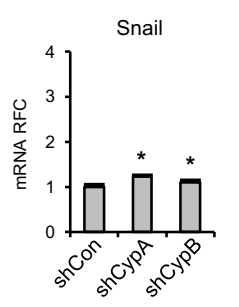

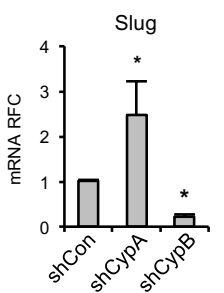
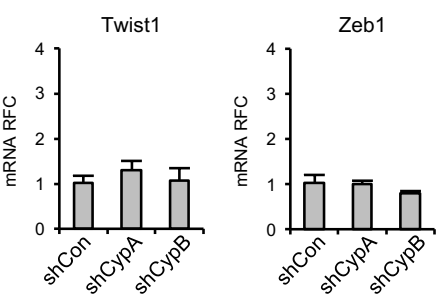

M

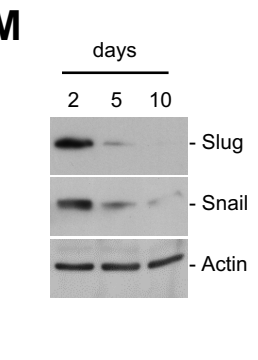

$\mathbf{N}$

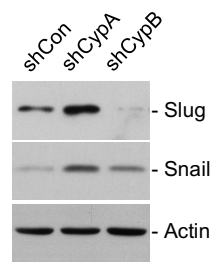


A

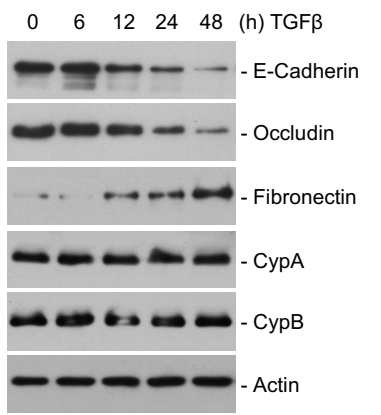

B

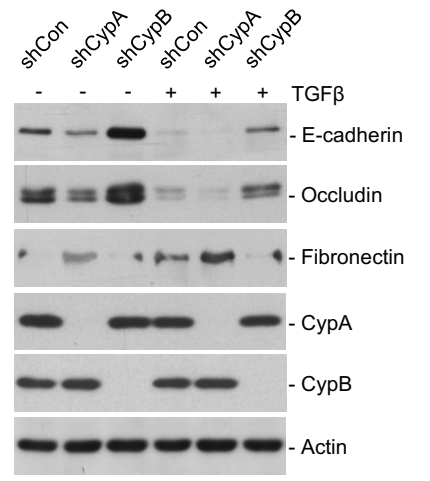

C

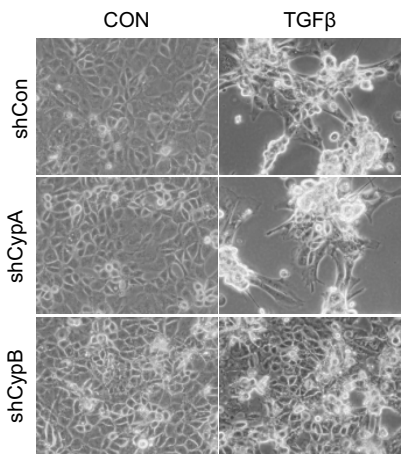

D

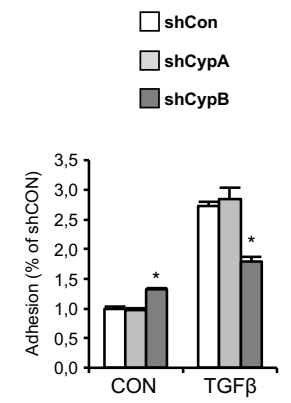

E

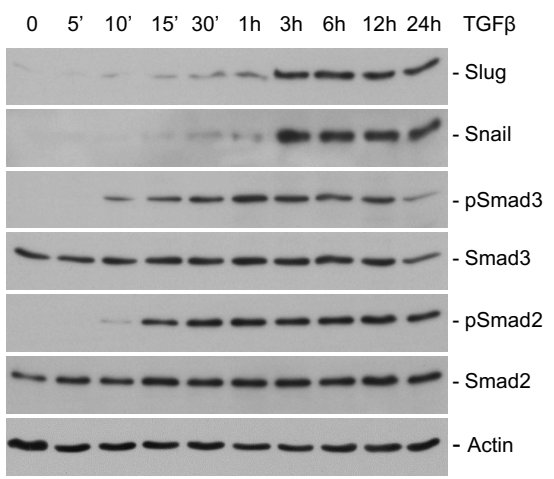

G

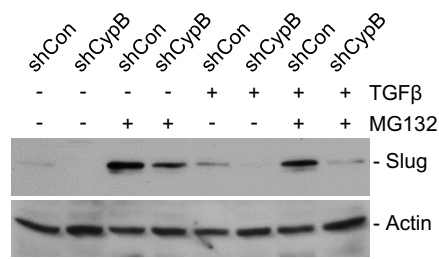

F
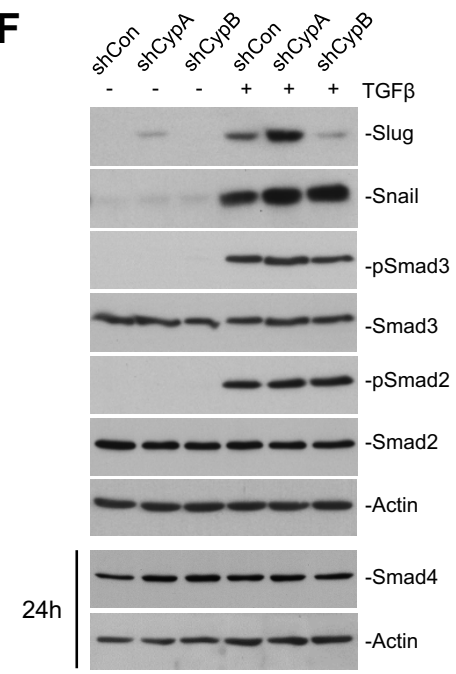

$\square$ shCon $\square$ shCypA $\square$ shCypB
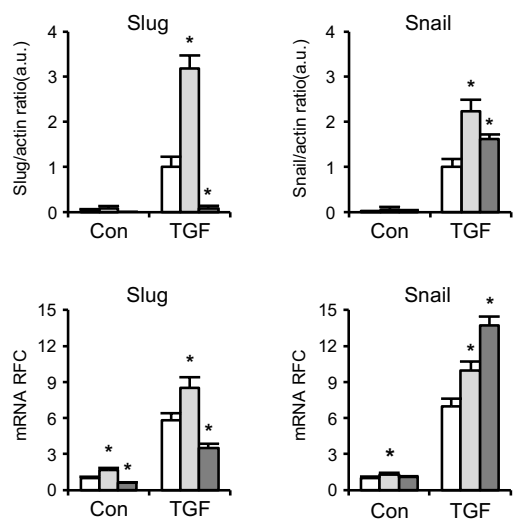
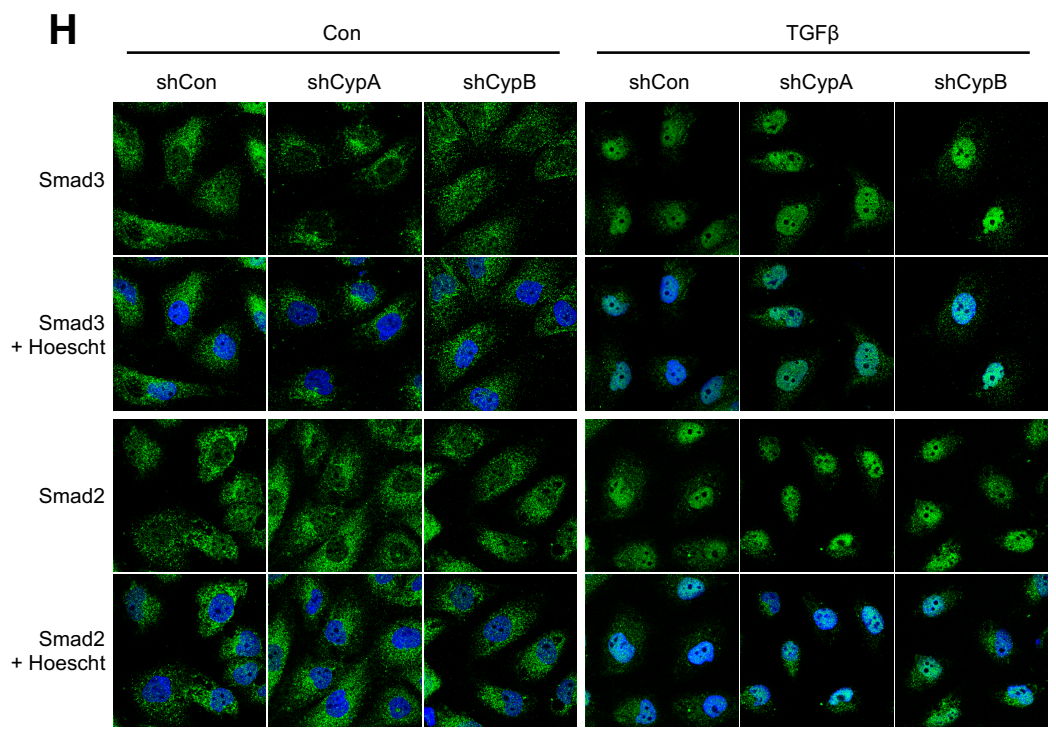

$\square$ shCon $\square$ shCypA $\square$ shCypB
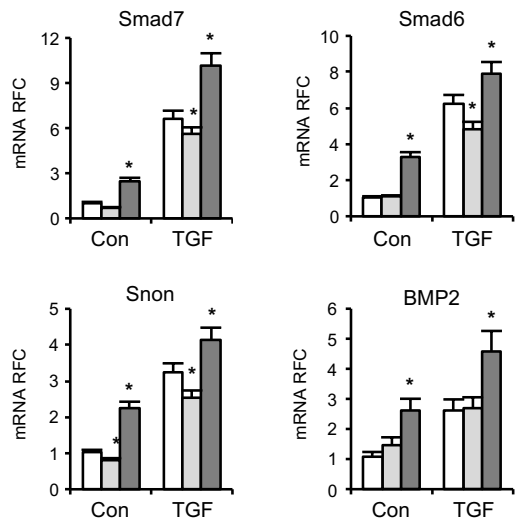
A

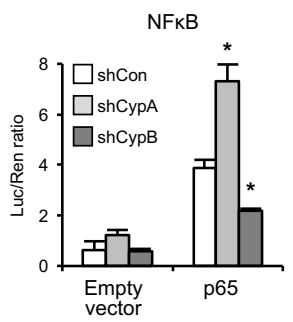

D

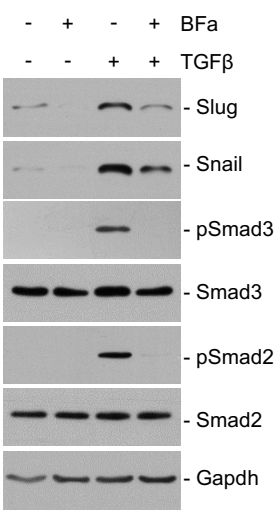

B

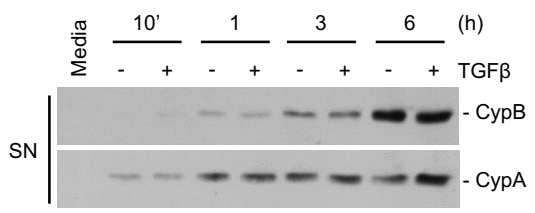

E

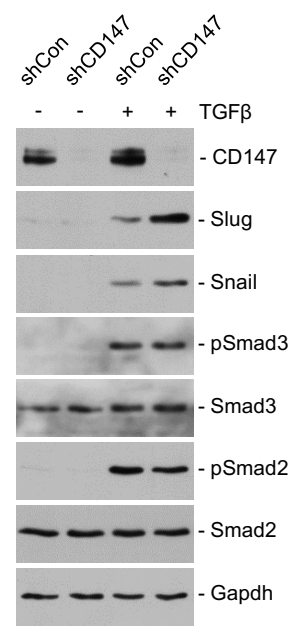

C

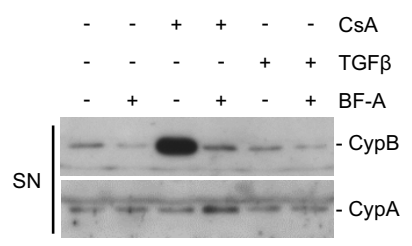

F

$\begin{array}{lllllllllll}0 & 12,5 & 25 & 50 & 100 & 0 & 12,5 & 25 & 50 & 100 & \operatorname{rCypB}(\mathrm{nM})\end{array}$

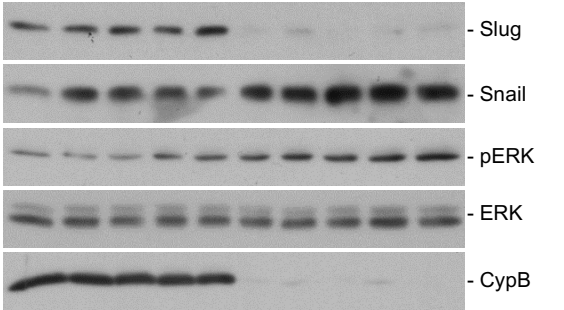




\section{Figure 4}

A
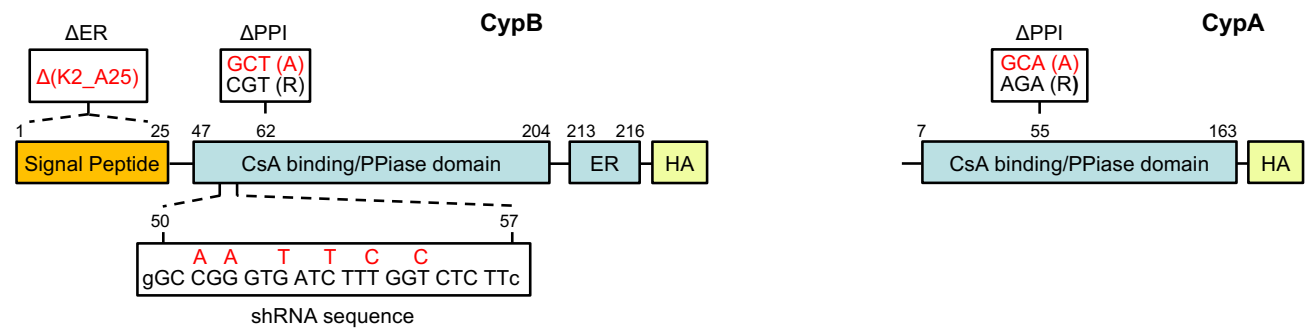

B

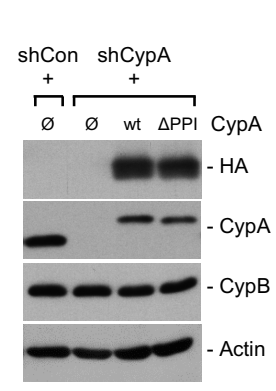

E

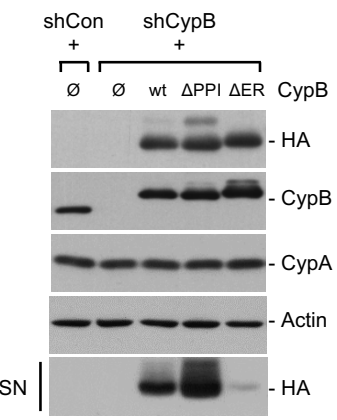

G

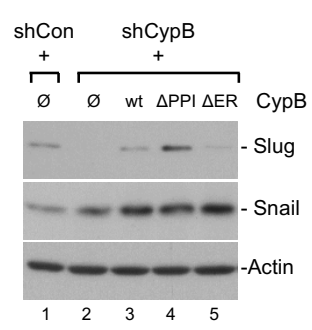

C

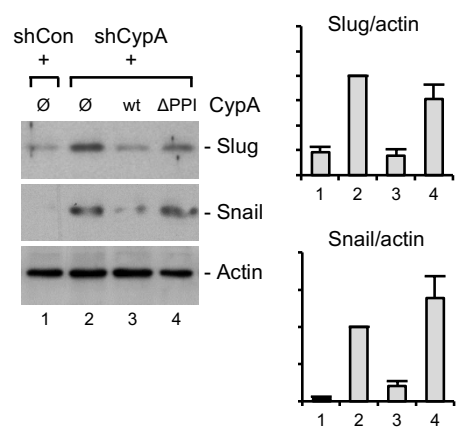

F

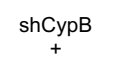

CypB wt

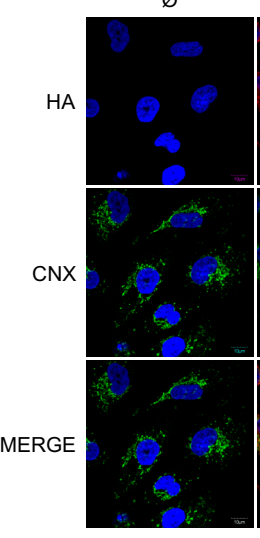

H

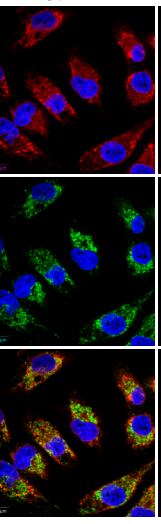

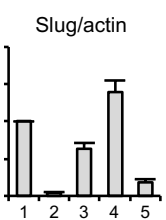

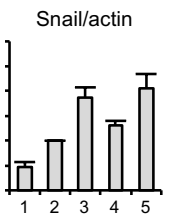

D shCon shCypA
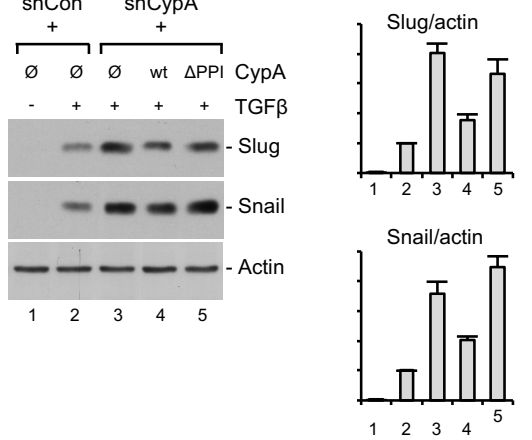

shCypB

shCypB shCypB

CypB $\triangle \mathrm{PPI}$

CypB $\triangle E R$

СурВ $\Delta \mathrm{ER}$
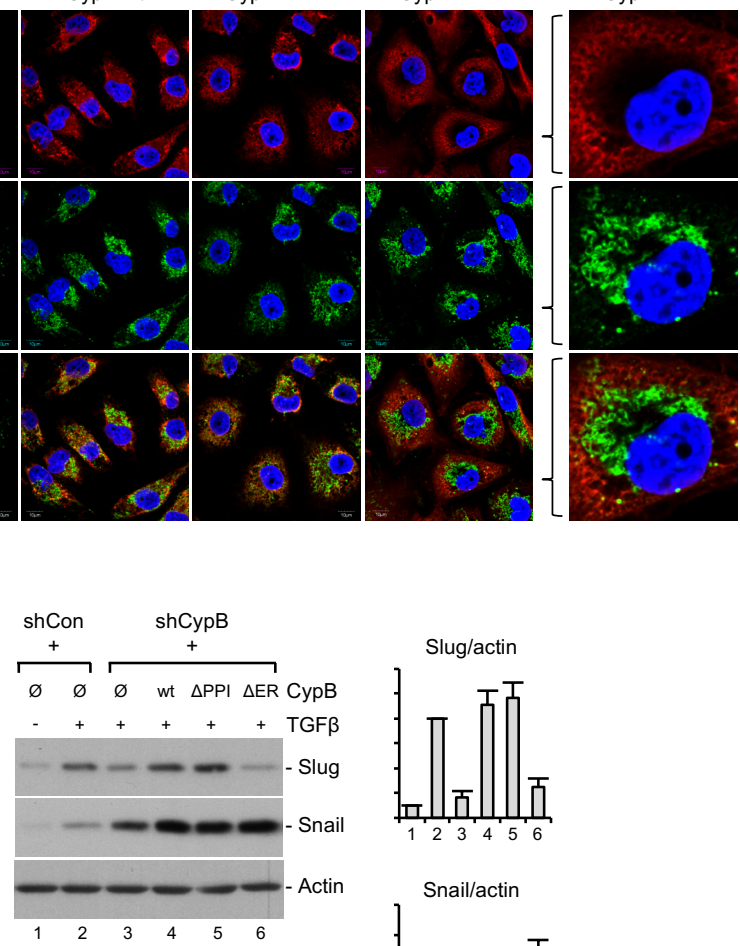

Slug/actin
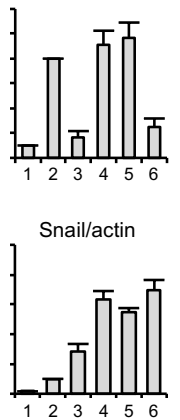
A

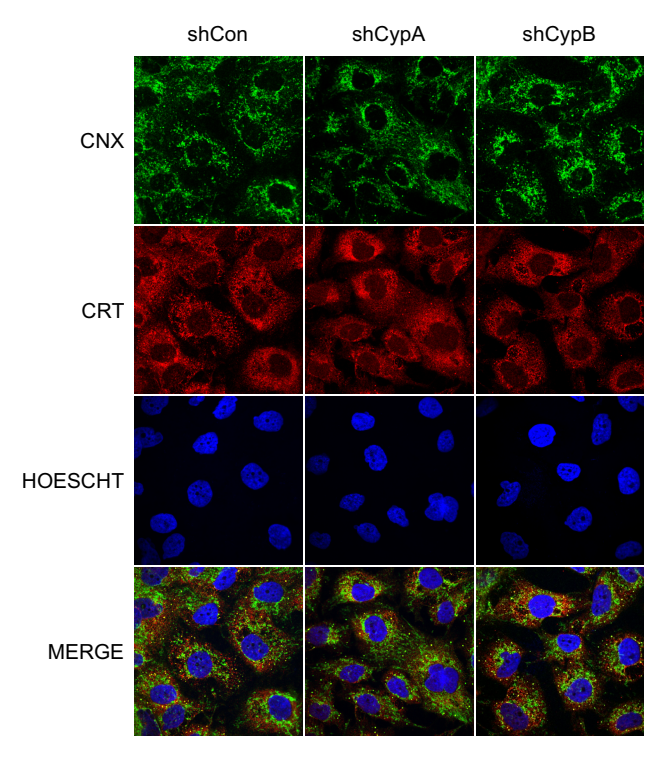

C

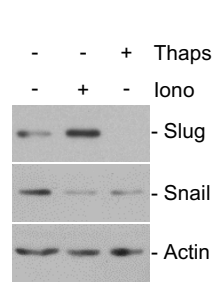

B

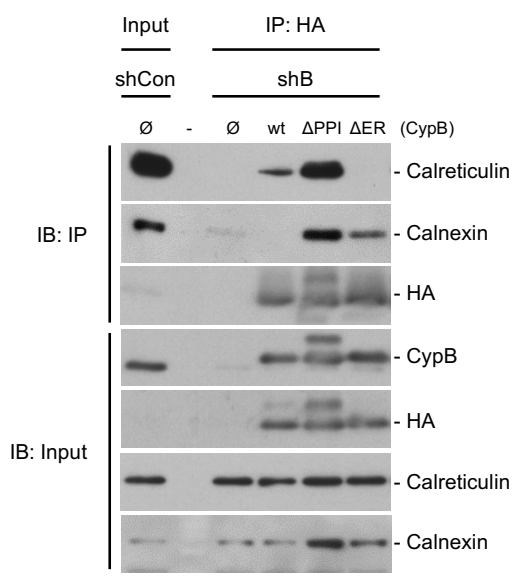

E

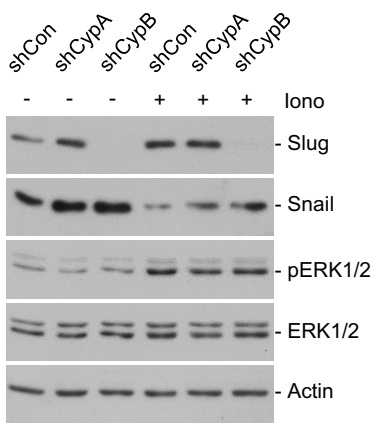




\section{Figure 6}

A

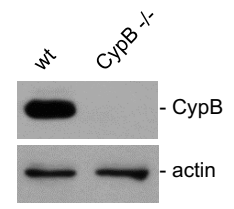

B

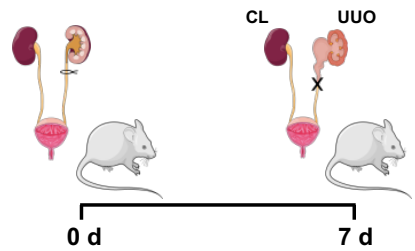

C

wt

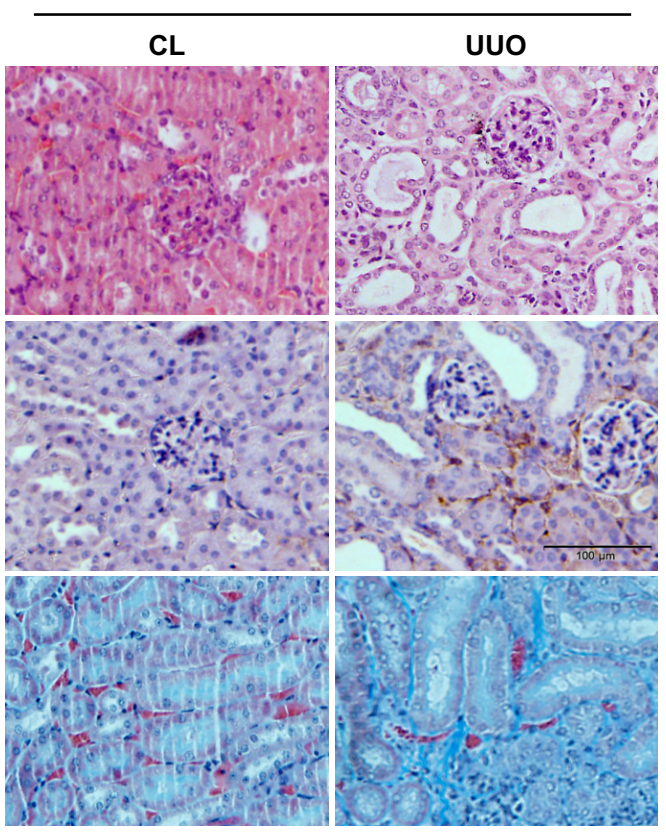

D

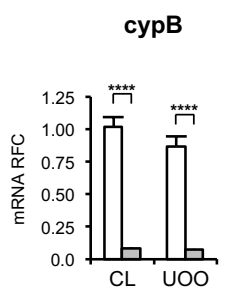

E
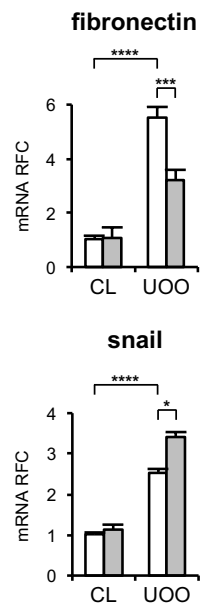

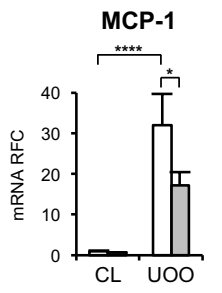

collagen-la

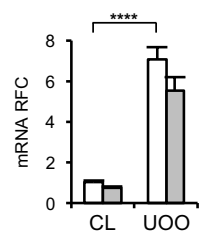

slug

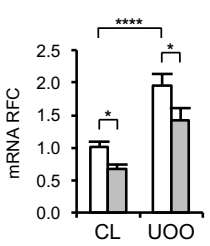

$\square$ wt
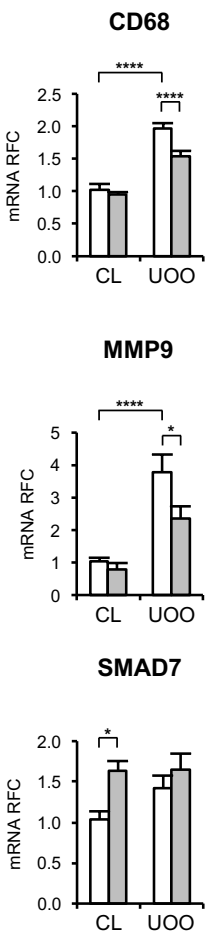

CypB -/-
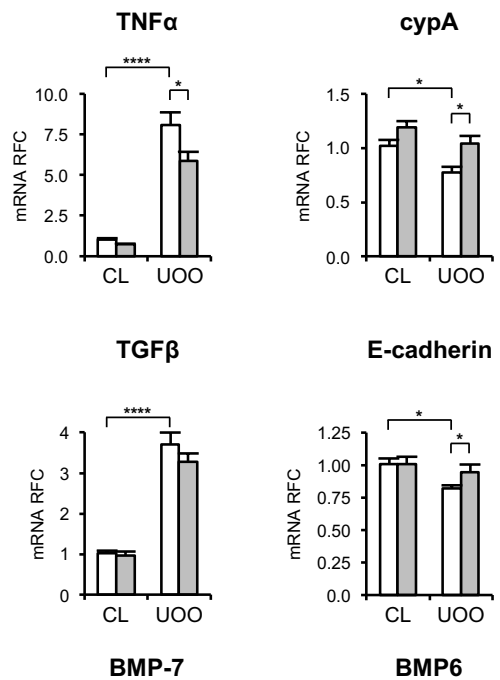

CD147
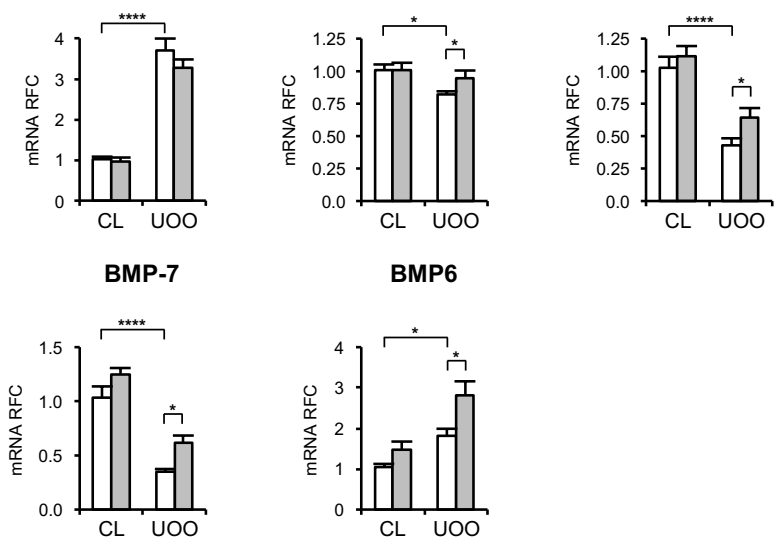

E-cadherin

BMP6

H\&E

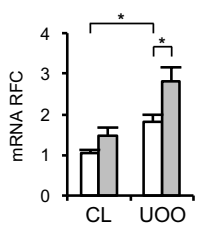




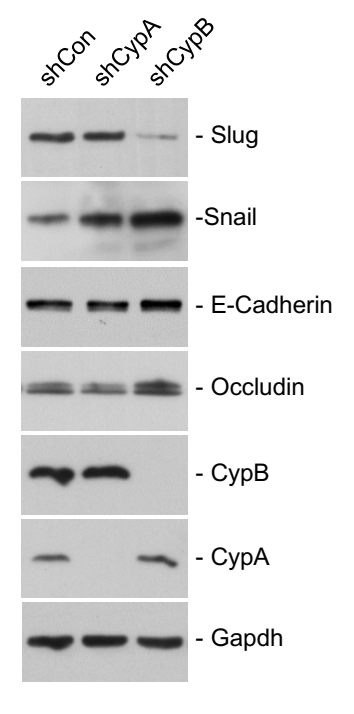

Figure Supp1 (RPTEC)

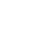

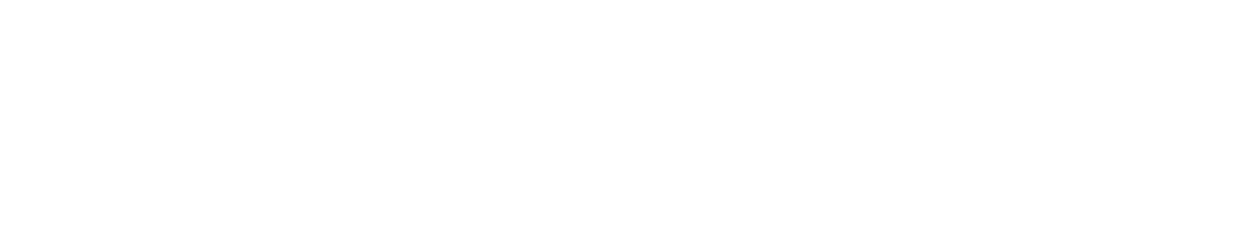
(1)

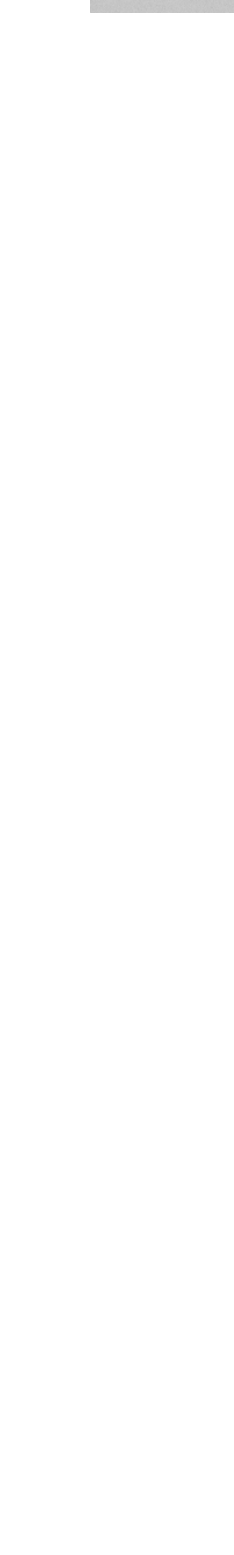

